Gerión. Revista de Historia Antigua

ISSN: 0213-0181

\title{
Politeia nella storiografia e nel pensiero storico greco tra V e IV secolo a.C.: la questione della continuità e del mutamento
}

\author{
Elisabetta Poddighe
}

Recibido: 19 de febrero de 2019 / Aceptado: 14 de junio de 2019

Riassunto. Nell'ambito della "grande" storiografia come della storiografia locale la riflessione sul tema della politeia è stata, fino ad Aristotele, prevalentemente svolta nel segno della continuità tra il passato della polis e il suo presente. Questo non significa che il tema della discontinuità costituzionale (metabole politeion) non abbia trovato spazio nell'opera degli storiografi, ma piuttosto che quel tema non è stato utilizzato per riflettere in un'ottica universale sui processi di cambiamento della storia sociale delle poleis. Questo sviluppo decisivo si deve alla ricerca storica aristotelica nella quale lo studio della politeia è messo al servizio di un modo nuovo di fare la storia della polis (civic historiography).

Parole chiave: politeia; storiografia locale; grande storiografia; intentional history; metabole politeion; storia universale; civic historiography; Aristotele.

\section{[en] Politeia in Classical Greek Historiography and in Historical Thought: Continuity and Change}

\begin{abstract}
Reflection on the politeia in both great and local historiography has been mainly focused on continuity between the past and the present of the polis. This does not mean that historiographers excluded the theme of constitutional discontinuity (metabole politeion) from their works, but rather that they never used it to adopt a universal perspective to consider the processes of change in the social history of the poleis. Such a decisive development came about thanks to Aristotle's historical research in which the study of the politeia is put at the service of a new way of shaping the history of the polis (civic historiography).

Keywords: Politeia; Local Historiography; Great Historiography; Intentional History; metabole politeion; Universal History; Civic Historiography; Aristotle.

Sumario. 1. Introduzione. 2. Storia e politeia fra "continuous history" e "static historiography": i casi di Atene e Sparta. 3. La metabole politeion nella riflessione storiografica e nel pensiero politico prima di Aristotele. 4. Il contributo di Aristotele: metabole politeion e storia della polis in una prospettiva universale. 5. Bibliografia.
\end{abstract}

Cómo citar: Poddighe, E. (2019): Politeia nella storiografia e nel pensiero storico greco tra V e IV secolo a.C.: la questione della continuità e del mutamento, en Gerión 37/2, 271-300.

\footnotetext{
1 Università degli Studi di Cagliari.

E-mail: poddighe@unica.it
} 


\section{Introduzione}

La politeia è la carta d'identità di una polis: ne identifica i tratti distintivi. Un'efficace definizione del rapporto fra una polis e la sua politeia è quella che dà Aristotele nel libro terzo della Politica: ${ }^{2}$ qui la polis è definità come una comunità (koinonia) di cittadini (politai) accomunati dalla costituzione (politeia). In greco, la definizione è

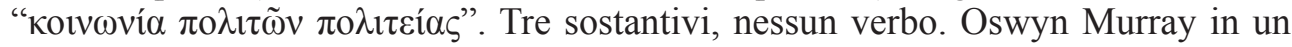
saggio del 1993 (Polis and Politeia in Aristotle) ha riconosciuto la pregnanza di questa definizione di difficile traduzione. ${ }^{3}$ Il senso è quello di una comunità di cittadini la cui comunanza si fonda sulla politeia. Resta nella sua essenzialità una delle definizioni che meglio mette a fuoco la capacità della politeia di tenere dentro tutta la polis: essa identifica non solo le sue istituzioni politiche e giuridiche, $\mathrm{i}$ corpi legislativi, l'ethos di una comunità, ${ }^{4}$ ma comprende tutto ciò che i cittadini hanno deciso di avere in comune, quel "comune interesse" che li rappresenta dentro la polis e fuori da essa. Sarebbe riduttivo intendere la politeia come "costituzione" nel senso moderno del termine. La politeia è la forma e l'essenza stessa della città.

Considerato il ruolo decisivo che la polis assegna alla sua politeia non stupisce che tanto spazio sia stato riservato a questo concetto nella riflessione storica greca. Attraverso la politeia può essere rievocata e raccontata la storia della polis. Ma quali sono i modelli di rappresentazione della politeia utilizzati nella storiografia e nel pensiero storico dei Greci?

Il primo obiettivo di questa disamina è provare a mettere a fuoco i modelli utilizzati per rievocare attraverso la politeia la storia della polis nella cosiddetta "grande" storiografia e nella storiografia locale. Al riguardo, le acquisizioni della ricerca consentono di isolare con relativa sicurezza alcuni aspetti del problema dei quali si darà conto in questo lavoro: (1) fino ad Aristotele è mancata nella storiografia greca una significativa interazione tra indagine storica e teoria politica; (2) tale assenza è all'origine di due caratteristiche dell'atteggiamento assunto riguardo alla politeia dagli storici sia panellenici che locali.

La prima caratteristica è rappresentata dal fatto che nel discorso storico la politeia serve a saldare passato e presente della storia della polis in un continuum che annulla e oblitera i processi di cambiamento. In questa rappresentazione della politeia ideologicamente orientata secondo un modello che gli studiosi definiscono "continuista" -comune alla grande storiografia e alla storiografia locale- si esprime il contributo dello storico alla narrazione della storia della polis o "civic historiography". Una seconda caratteristica -che discende dalla prima- riguarda l'inadeguatezza dei tentativi compiuti prima di Aristotele di usare il tema dell'instabilità costituzionale (metabole politeion) per spiegare i cambiamenti storici della polis in un'ottica di storia universale. Un modo radicalmente nuovo di riflettere sulla politeia per ricostruire la storia sociale della polis si riconosce nella ricerca storica di Aristotele. Al carattere originale della sua riflessione sono dedicate le pagine conclusive di questo lavoro.

Per cominciare, un excursus -necessariamente schematico- sulla grande storiografia e sulla storiografia locale.

\footnotetext{
Arist. Pol. 1276 b 1-2.

Murray 1993a, 197-210.

Arist. Pol. 1289a 15-18.
} 


\section{Storia e politeia fra "continuous history" e "static historiography": i casi di Atene e Sparta}

Innazitutto, in ordine di importanza e di rilevanza nella storia degli studi, è la cosiddetta "grande storiografia". ${ }^{5}$ Consacrata alla narrazione dei grandi conflitti combattuti nel corso del V secolo per l'egemonia sui Greci era perciò definita "Great History" da Felix Jacoby che con questo attributo la contrapponeva alla storiografia locale. " "Grandi" erano per gli storiografi Erodoto e Tucidide le azioni (praxeis) delle quali si impegnavano a dare una descrizione: grandi in rapporto alle dimensioni del conflitto (le guerre persiane, la guerra del Peloponneso) e all'ampiezza dell'area geo-politica coinvolta. ${ }^{7}$ La grande storiografia comprendeva la storia di singole poleis, e non solo di poleis quali Atene e Sparta, come è noto. ${ }^{8}$ Ma è ai casi di Atene e Sparta che qui si farà riferimento, pur nella consapevolezza del quadro parziale che la relativa documentazione consente di ricostruire, perché sono i casi che conosciamo meglio e che rivelano le modalità secondo le quali gli storiografi del V secolo hanno utilizzato il tema della politeia nel loro discorso storico.

In entrambi i casi, possiamo contare su acquisizioni della ricerca consolidate: l'evocazione della politeia opera nell'uno e nell'altro caso entro una prospettiva continuista. Meglio noto il caso ateniese. Nel V secolo gli storici greci hanno scelto di riflettere sulla politeia democratica di Atene, dunque sugli strumenti concettuali elaborati da quella democrazia, sulle prerogative della cittadinanza democratica, in termini di valori assoluti. Non hanno cioè voluto rappresentare la politeia di Atene come l'esito di un lungo processo storico che inizia al principio del VI secolo e si compie a metà del V. ${ }^{9} \mathrm{E}$ un atteggiamento che Diego Lanza ha considerato in rapporto alla "necessità di credere in un'Atene da sempre democratica nella quale l'uguaglianza dei cittadini risalirebbe al tempo delle origini" ${ }^{10}$ con ciò che ne consegue sul piano della rappresentazione storiografica dell'evoluzione della democrazia ateniese. Difficile non essere d'accordo con quanto, a proposito di questo stesso atteggiamento, ha osservato Mark Munn "tradition held that democracy was an Athenian trait, and therefore it must have been so from the time of its legendary founding hero. It was not useful to have history correct that tradition". ${ }^{11}$ La storia insomma non doveva "correggere" l'idea tradizionale di politeia.

L'assenza di un vero interesse per il tema dell'evoluzione della politeia rispetto al quadro delle origini è un dato che già aveva messo a fuoco Jacqueline Bordes in uno studio del 1982 che affronta nel modo più sistematico la storia di quel concetto nel pensiero greco. ${ }^{12}$ Dunque, gli storici del V secolo disegnano il profilo storico della politeia ateniese nel segno di quella sostanziale continuità che gli studiosi definiscono la continuous history o continuous story di Atene. ${ }^{13}$

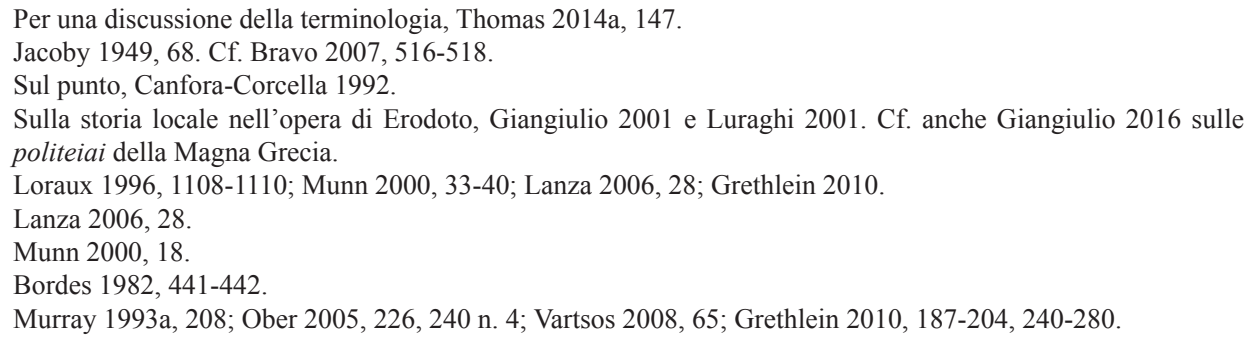


Si possono fare pochi esempi del modo continuum con cui gli storici greci hanno rappresentato il carattere della politeia ateniese. Indicativo è l'atteggiamento di Erodoto nel quinto libro delle Storie. Per Erodoto esiste una sola politeia di Atene, è quella democratica, ed è una politeia democratica che non ha conosciuto sviluppi significativi dopo la fase delle sue origini. ${ }^{14}$ Quell'idea democratica si definisce in contrapposizione alla tirannide ed è appiattita sulla riforma di Clistene. ${ }^{15}$ Erodoto non ha interesse ad affrontare il problema storico della metabole politeion introdotto dal nuovo regime democratico, anche se lo identifica e lo racconta, e non descrive gli organi decisionali democratici operanti nelle politeiai che hanno preceduto e seguito la riforma di Clistene. Né il racconto di come Clistene sia arrivato a riformare la politeia ateniese sviluppa una riflessione attenta alle dinamiche socio-economiche di Atene, e questo anche se Erodoto racconta dei nuovi diritti allargati al demos con riguardo all'accesso alle funzioni politiche: ${ }^{16}$ manca infatti un'adeguata trattazione del contesto socio-economico nel quale la riforma della politeia è stata realizzata. ${ }^{17}$ Erodoto inoltre non considera l'evoluzione delle istituzioni democratiche: tace sul funzionamento dei tribunali popolari e dell'assemblea prima e dopo la riforma del 508, né mostra un particolare interesse a descrivere l'ordine costituzionale democratico in rapporto alle procedure di selezione dei magistrati (tanto che quando ne dà conto commette degli errori). ${ }^{18}$ Per Erodoto, la politeia democratica di Atene è "una", nasce con Clistene e il suo carattere costitutivo si risolve nel fatto di incarnare l'esatto opposto della tirannide. ${ }^{19}$

L'atteggiamento di Tucidide è ugualmente rappresentativo della scarsa propensione a sviluppare una riflessione rivolta alla storia della politeia di Atene nelle sue diverse fasi. Non c'è interesse per l'evoluzione nel lungo periodo ma solo per quelli che Kurt Raaflaub ha chiamato i "momentous events" della storia di un regime politico. ${ }^{20} \grave{E}$ un atteggiamento che già Andrew Lintott aveva messo a fuoco nel suo pionieristico lavoro sulla stasis del $1982 .{ }^{21}$ L'approccio col quale Tucidide riflette sulle modifiche che hanno interessato la politeia ateniese è quello che rivela nell'ottavo libro della sua opera il famoso giudizio sull'esperimento di governo dei Cinquemila nel 411: quella breve fase costituzionale durante la quale la pienezza dei diritti politici fu riservata a un corpo di cinquemila cittadini selezionati sulla base del censo (ossia capaci di armarsi a proprie spese). Al riguardo Tucidide si limita a osservare che quel regime ha rappresentato "la prima forma di buon governo che gli ateniesi si siano dati, almeno ai miei tempi", ${ }^{22}$ evitando di sviluppare un giudizio più articolato che valutasse quella forma di politeia in rapporto alle epoche più remote così come a quelle più recenti. ${ }^{23}$ Né qui Tucidide -come ha mostrato Laura Sancho

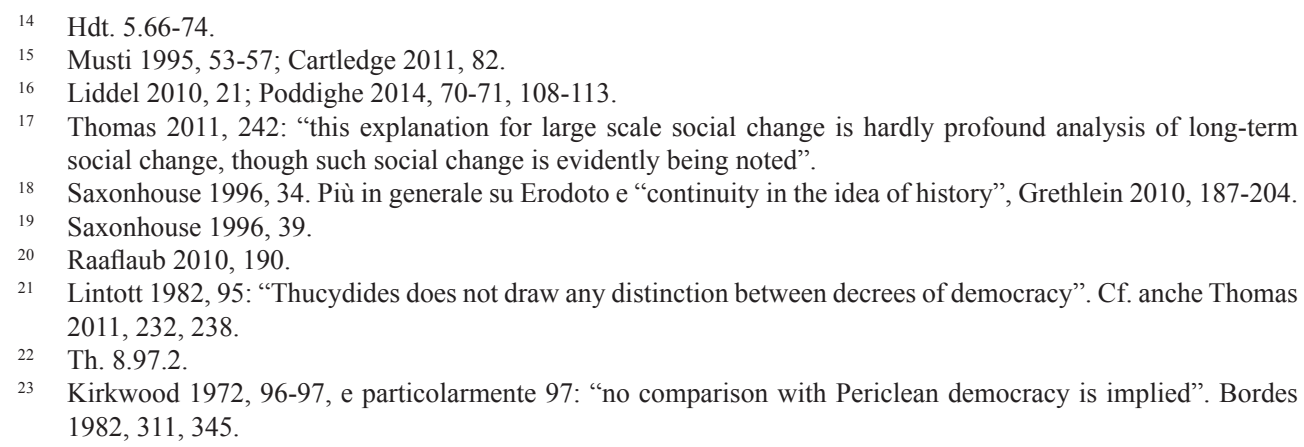


Rocher-sviluppa una teorizzazione di un modello moderato di politeia del tipo della costituzione mista. ${ }^{24}$

Diversamente, come rileva Luciano Canfora, "Tucidide non si esprime sulla migliore forma politica in assoluto" 25 e limita il giudizio all'esperienza dei suoi tempi; così "questo pensiero, che pure parrebbe rilevante nel quadro dell'acceso dibattito costituzionale in Atene, non ha grandi sviluppi e viene subito abbandonato". ${ }^{26}$ È chiaro che Tucidide riconosce il cambiamento istituzionale. ${ }^{27}$ Sceglie però di non portare la sua riflessione sul piano teorico ${ }^{28}$ e la politeia è evocata con riferimento esclusivo a quel "momentous event".

Sebbene, dunque, i sommovimenti istituzionali di Atene siano stati riconosciuti e raccontati dagli storici, è chiaro che questi ultimi non hanno voluto affrontare il tema del cambiamento costituzionale come problema in sé ovvero non hanno voluto affrontare sul piano teorico il problema storico del mutamento della politeia. ${ }^{29}$

Nell'ambito di questa rappresentazione statica della politeia ateniese, sono tra $\mathrm{i}$ modelli retorici privilegiati i discorsi funebri o logoi epitaphioi, che celebrano i caratteri costitutivi della politeia ateniese, dello stile di vita, dei modi di fare e pensare (tropoi) dei cittadini ateniesi. ${ }^{30} \mathrm{Il}$ discorso funebre pronunciato da Pericle per i caduti del primo anno della guerra peloponnesiaca, nel 431, è un esempio di questo genere. È noto nei termini in cui lo riferisce Tucidide nel secondo libro della sua opera, la Guerra del Peloponneso e contiene la più rappresentativa descrizione del carattere della politeia democratica ateniese. ${ }^{31}$ Nel lungo discorso che Tucidide fa pronunciare a Pericle $\mathrm{e}^{32}$ è la politeia il tema a partire dal quale è possibile rievocare $\mathrm{i}$ caratteri propri degli Ateniesi, le regole che gli Ateniesi hanno scelto di darsi in materia di giustizia e i criteri secondo i quali essi regolano l'accesso alle cariche pubbliche. Trattandosi di una politeia democratica, l'accento è posto dal Pericle tucidideo sul principio che sono i molti e governare, che la legge tutela l'uguaglianza e che la povertà non impedisce al meritevole di accedere alle cariche. ${ }^{33} \mathrm{La}$ specificità della politeia democratica ateniese è richiamata non solo a livello politicoistituzionale, ma anche sul piano dei valori etici condivisi. È indicativo che ci sia un richiamo alle leggi scritte degli Ateniesi contro ogni ingiustizia e insieme ci sia l'evocazione delle leggi non scritte, che non sono leggi universali o divine, ma sono invece le leggi che trovano applicazione ad Atene in quanto espressione del carattere degli Ateniesi, in quanto valori condivisi dalla comunità.

Tutto ciò che gli Ateniesi hanno deciso di condividere sul piano della politica e della coesione sociale è la loro politeia. Si tratta, come è evidente, di materiale utilissimo a riflettere sulla politeia ateniese, e in particolare sui modelli della

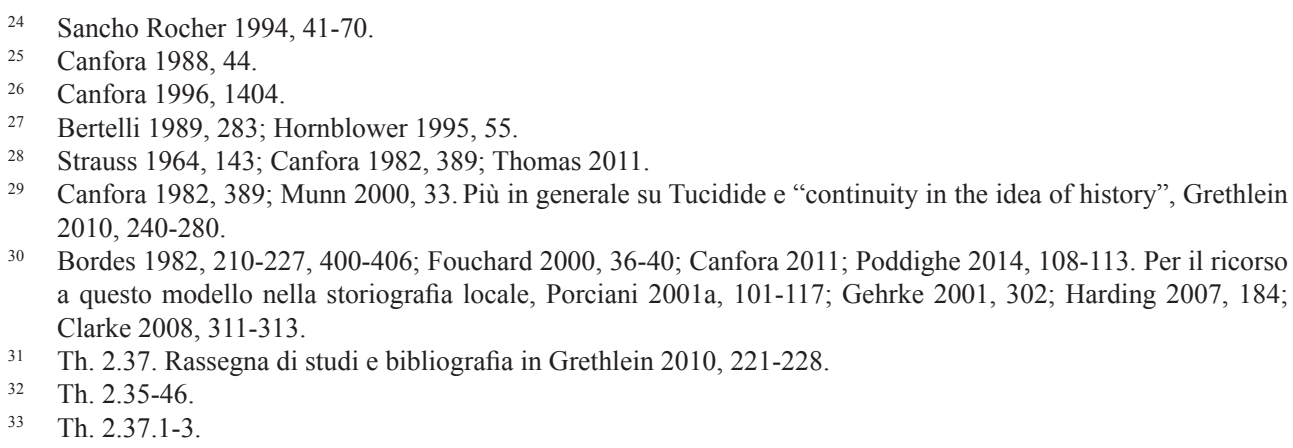

30 Bordes 1982, 210-227, 400-406; Fouchard 2000, 36-40; Canfora 2011; Poddighe 2014, 108-113. Per il ricorso a questo modello nella storiografia locale, Porciani 2001a, 101-117; Gehrke 2001, 302; Harding 2007, 184; Clarke 2008, 311-313.

31 Th. 2.37. Rassegna di studi e bibliografia in Grethlein 2010, 221-228.

2 Th. 2.35-46.

33 Th. 2.37.1-3. 
cittadinanza democratica ateniese, ma trattato in modo statico, ovvero senza riguardo per lo sviluppo storico delle acquisizioni democratiche. ${ }^{34}$ Nei discorsi funebri la storia della politeia è convenzionalmente asservita agli scopi celebrativi di un genere retorico la cui funzione è dare una "auto-rappresentazione ideologica della città". ${ }^{35} \mathrm{I}$ logoi epitaphioi servono a rievocare il carattere della democrazia, a definire lo stato della sua politeia, senza dare conto dell'evoluzione subita dalle sue istituzioni. ${ }^{36}$ Serve a dire "noi siamo così" e siamo così "da sempre".

Il rapporto col passato istituzionale si svolge a partire da un "limited set of inexorable themes" senza preoccupazione alcuna per lo svolgimento cronologico né per la comprensione storica. ${ }^{37} \mathrm{E}$ la sommaria rievocazione di temi "triti" che "fanno a pugni con la realtà effettuale" 38 e che si svolge in un continuum di spazio e tempo che annulla la dimensione storica. ${ }^{39}$ E quello che Grethlein ha chiamato "the eternal continuum of the polis". ${ }^{40}$

La prospettiva continuista sulla politeia ateniese non rappresenta una prerogativa esclusiva dell'impostazione storiografica. Lo stesso atteggiamento è riconoscibile nella retorica deliberativa e giudiziaria ${ }^{41}$ così come nella letteratura pamphlettistica. Anche nello scritto pseudo senofonteo sulla politeia degli Ateniesi, il regime descritto non è mai considerato rispetto al suo sviluppo storico: nessuno sguardo è rivolto alla politeia delle origini, ${ }^{42}$ nessun rilievo è dato al tema delle origini della talassocrazia, che pure è il fattore storico che "fonda" l'impero ateniese ed è tra i nuclei tematici principali dell'intero scritto. ${ }^{43}$

La prospettiva continuista rappresenta il modo ateniese di rappresentare il passato costituzionale della città, la storia della sua politeia. ${ }^{44}$ Il ruolo sociale che questo atteggiamento assume si esprime nella possibilità di "counteract contingency by establishing regularity": un aspetto che è stato bene riconosciuto da Jonas Grethlein nei suoi numerosi studi sul ruolo che nella "forma" narrativa degli storici greci ha assunto il tema del continuum. ${ }^{45}$

Della possibilità di saldare passato e presente della polis in un continuum la politeia era strumento essenziale. La politeia ateniese era rievocata per unire, nel tempo e nello spazio, soprattutto quello sociale. ${ }^{46}$ Nella grande storiografia, alla funzione sociale della cosiddetta civic historiography assolve perciò la concezione di una politeia ateniese da sempre uguale a se stessa. E ciò perché la politeia serve a

\footnotetext{
Grethlein 2010, 108-113; 2013a, 127-134.

Canfora 2011, 9.

Loraux 1993; Grethlein 2010, 108-113.

Munn 2000, 36-40; Clarke 2008, 311-313; Grethlein 2010, 108-113; 2013a, 132-134.

Canfora 2011, 5-24

Grethlein 2010, 105-125; 2013a 127-134.

40 La ragione, secondo Grethlein $(2010,115-117)$, è che quel continuum "overcomes the personal experience of contingency of chance" in quanto "erases the borderlines between different stages of the past" (115).

41 Cf. ora Canevaro 2017 e Barbato 2017.

42 Bordes 1982, 162: “le Pseudo-Xénophon ne conçoit pourtant pas qu' il puisse y avoir plusieurs formes de démocratie; il ne se préoccupe nullement d'un passé institutionnel différent du présent". Così anche Lenfant 2018 .

43 Bordes 1982, 139-163.

44 Per l'evocazione della politeia secondo modelli analoghi nei tragici, Poddighe 2014, 111-112.

45 Grethlein 2011, 261: "Past and present were often linked by the idea of continuity". Cf. anche Grethlein 2010; 2012; 2014b, 245.

46 Per l'idea che la sua rievocazione oscurasse le differenze sociali all'interno della comunità civica: Ober 1989, 291; Epstein 2011, 90-93; Susanetti 2015, 7; Lenfant (c.s.).
} 
dare una rappresentazione ideologica della città piuttosto che a ricostruire e a raccontare la storia sociale della polis. ${ }^{47}$

Ora, la prospettiva continuista che appare comune alla ricostruzione di Tucidide ed Erodoto, quando affrontano questioni collegate alla storia della politeia ateniese, è stata al centro di studi che hanno provato a spiegarne l'origine, non solo in rapporto alla funzione ideologica, ma anche in relazione al carattere della ragione storica e culturale greca. ${ }^{48}$

Tra le riflessioni più sistematiche è quella di Christian Meier, il quale riconosce nel sapere storico greco del V secolo i caratteri che si sono opposti alla "comprensione" e alla sistemazione "lungo un fronte davvero ampio" dei processi di mutamento costituzionale.$^{49}$ Il dato saliente è, secondo Meier, che nella Grecia classica sono stati descritti dagli storici soprattutto processi di breve periodo,${ }^{50}$ nei quali il cambiamento appariva "troppo debole per essere in grado di varcare la soglia che si opponeva alla sua comprensione". ${ }^{51}$ A rendere più difficoltoso il superamento di quella soglia sarebbe, secondo Meier, un carattere costitutivo della ragione storica del V secolo che faceva oggetto della sua indagine più l'agire che il cambiare, i fatti e gli eventi stessi più che "il cambiare, il mutamento". ${ }^{2}$ Perciò, sebbene Erodoto o Tucidide o altri scrittori greci del V secolo abbiano "presunto" l'esistenza del cambiamento, nella loro narrazione storica il mutamento non appare studiato in quanto tale, in quanto cioè distaccato da chi lo ha causato, e quale "centro di gravità" dell'indagine storica. ${ }^{53} \mathrm{Gli}$ scrittori greci del V secolo hanno "raccontato" la contrapposizione politica, il sommovimento a essa conseguente, e anche la singolarità del nuovo corso istituzionale, non hanno però dato dignità teorica al tema del mutamento della politeia.

Altri studiosi hanno messo in relazione l'atteggiamento degli storici nei confronti del mutamento della politeia con la generale resistenza culturale dei Greci di fronte all'innovazione e al cambiamento. È noto, infatti, che nei diversi campi di sapere, da quello filosofico a quello medico-scientifico a quello tecnico-artistico, le rare posizioni espresse a favore dell'innovazione "utile" sono rimaste -tra V e IV secolo- isolate e all'ombra di un pensiero dominante ostile all'idea del mutamento e alla ricerca del nuovo.$^{54}$ Lo stesso pensiero scientifico che, nel $\mathrm{V}$ secolo, ha sviluppato le riflessioni più nette in tema di innovazione,,$^{55}$ ha teorizzato come sola forma di innovazione utile quella contemplata da un sapere di tipo cumulativo e quantitativo ${ }^{56}$ in cui l'esperienza presente si somma a quella passata senza soluzione di continuità. ${ }^{57} \mathrm{Se}$ si guarda alla storia degli studi, insomma, pur in presenza di divisioni tra gli studiosi riguardo al peso che assumono le singole posizioni espresse a favore della ricerca del "nuovo", appare consolidata l'opinione che i Greci siano stati nel corso dell'intera loro storia poco interessati a sviluppare sia il concetto di innovazione che quello di rivoluzione $\mathrm{e}^{58} \mathrm{e}$ siano

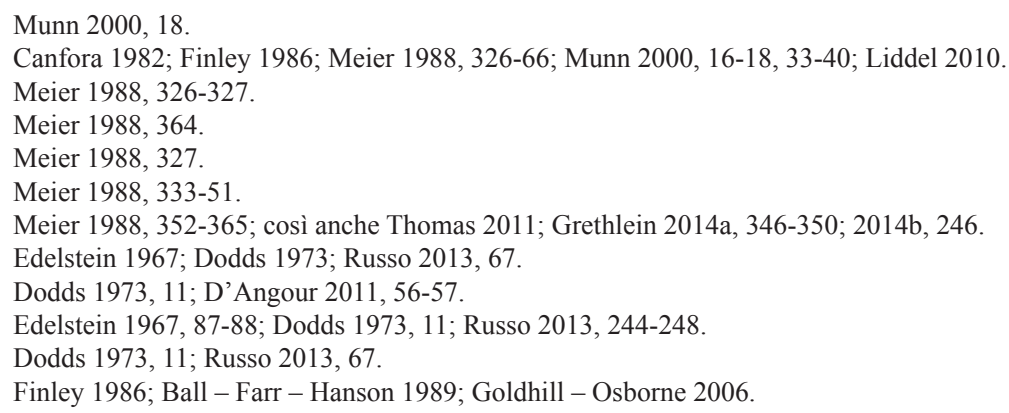


stati tendenzialmente ostili al cambiamento che non fosse circoscritto all'ambito delle tecniche e dei saperi scientifici. È significativo che un rapporto di dipendenza fra gli esiti di questo atteggiamento culturale e lo spazio che gli storici del V secolo hanno assegnato al tema dei rivolgimenti della politeia sia stato riconosciuto nella definizione che Tucidide $^{59}$ ha dato della (eversiva) ricerca di novità (neoteropoiia) degli Ateniesi, così temuta dagli Spartani: ricerca di novità che -secondo alcuni studiosi- andrebbe intesa come un riferimento alle riforme costituzionali di Efialte. ${ }^{60}$

Dunque, da un lato la funzione ideologica assegnata alla prospettiva continuista nella storiografia greca, dall'altra il carattere della ragione storica greca, anzi della cultura greca in generale, relativamente al tema del mutamento, possono aver concorso a determinare i modelli di rappresentazione della politeia ateniese nella grande storiografia del V secolo.

Il caso della politeia spartana è affrontato in modo analogo. Rappresentazioni storiografiche della immutabilità spartana si trovano in Erodoto e in Tucidide. ${ }^{61}$ Entrambi identificano nella riforma di Licurgo (o comunque nella fase storica in cui viene collocata la sua riforma) ${ }^{62}$ l'evento storico a partire dal quale cessano i rivolgimenti politici che seguirono alla fondazione di Sparta ad opera dei Dori.

L'idea che la fase stasiotica fosse pre-licurgica opera stabilmente nella grande storiografia tra V e IV secolo. ${ }^{63} \mathrm{Il}$ modello della ricostruzione storiografica è quello che gli storici chiamano "static historiography". Questa dà della storia di Sparta una visione statica appunto: crea un evento fondante (la politeia di Licurgo) e cristallizza quel momento iniziale che agisce come un momento "flash". ${ }^{64}$ Lo sviluppo delle istituzioni politiche, svoltosi storicamente fino alla fine del V secolo, contrasta con l'immagine di Sparta "fossilized". ${ }^{65}$ una città il cui assetto istituzionale è frutto di una vicenda storica che si esaurisce nella figura del suo mitico legislatore e risolve la sua storia nella politeia di Licurgo. ${ }^{66}$ Nino Luraghi ha applicato perciò al caso spartano la definizione di LeviStrauss di una "cold society", ovvero di una comunità politica che "has, in fact, excluded historical change as a means of continuity" ${ }^{67}$ "For a Spartan, to write the history of the city of Sparta was to write about the politeia of Lykourgos", scrive Daniel Tober a proposito di quel modello storiografico che finisce col condizionare inevitabilmente anche la storiografia locale la quale, lo vedremo, ugualmente non sviluppa una riflessione sulla politeia che vada oltre l'esposizione della politeia introdotta da Licurgo. ${ }^{68}$

Dunque, nella grande storiografia di $\mathrm{V}$ secolo, tratti distintivi e comuni dell'atteggiamento degli storici, quando utilizzano il tema della politeia per riflettere sulla storia delle poleis, sono l'assenza di uno sviluppo teorico del tema del cambiamento (o metabole) della politeia e l'assenza di segmenti di narrazione ampi

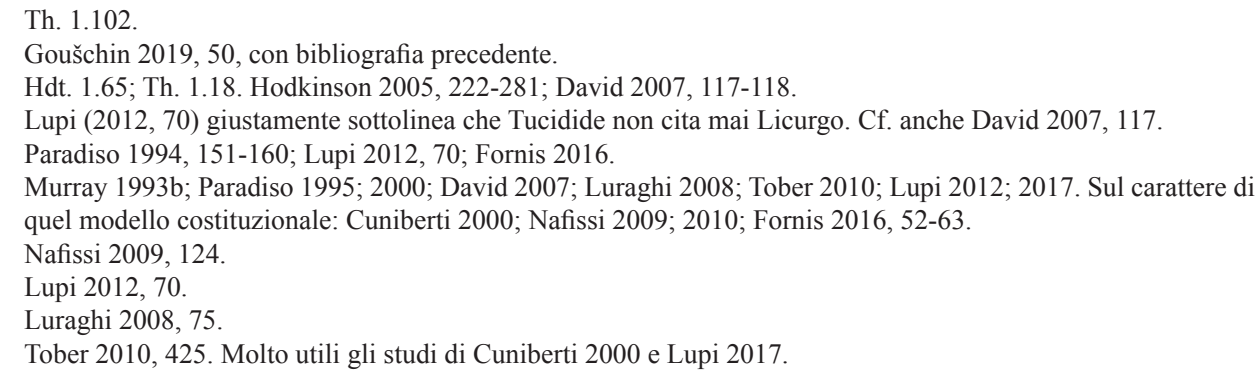


nei quali collocare il tema: a fare la storia della politeia sono i "momentous events" e il "flash" come mostrano rispettivamente i casi di Atene e Sparta.

Questi modelli storiografici del V secolo hanno condizionato la storiografia locale che conosciamo meglio, quella ateniese e spartana, ma, prima di passare a considerare i caratteri di questa variegata produzione storiografica, si deve dar conto dei modelli storiografici che sul tema della politeia sono riconoscibili nella "grande" storiografia di IV secolo. Anche in questo caso si registra una certa sintonia fra gli studiosi rispetto al fatto che gli storici antichi sembrano avere adottato forme del discorso storiografico compatibili con la storiografia del V secolo, e ciò sia per quanto riguarda l'attenzione riservata ai "momentous events" sia riguardo all'adozione di una prospettiva continuista, come indicano gli studi recenti di Rood e Grethlein, rispettivamente condotti sulle Elleniche ${ }^{69}$ e sull'Anabasi. ${ }^{70}$ Ugualmente riconosciuto negli studi recenti di Carol Atack su Senofonte (e su Isocrate) il ricorso a rielaborazioni della storia passata che attraverso la politeia aveva il fine di oscurare i cambiamenti. La studiosa inglese definisce come tratto distintivo e costante ("a continuing feature") del "politeia writing" sia in Senofonte che in Isocrate proprio l'oscuramento del tema del cambiamento, un oscuramento che opera a largo raggio, non essendo limitato alla politeia ateniese ma anche ai casi spartano e persiano. ${ }^{71}$ Un tratto già bene evidenziato dagli studiosi a proposito del trattato sulla politeia dei Lacedemoni scritta da Senofonte: qui infatti, anche se l'interesse dello storico è rivolto allo stile di vita più che al funzionamento istituzionale, la continuità con il modello della politeia di Licurgo è evidente così come la sua funzione di modello per il presente. ${ }^{72}$

Affermare che la prospettiva continuista è stata dominante nella storiografia di $\mathrm{V}$ e IV secolo non significa naturalmente negare che gli storici abbiano in diversi casi registrato e provato a spiegare il mutamento di alcune politeiai storiche. Il tema è, semmai, che tale processo di cambiamento, quando registrato e spiegato, assume irrimediabilmente la forma di una decadenza del sistema: è il mutamento inteso come allontanamento dal modello perfetto delle origini. Un dato significativo che, a mio avviso, rivela la forza del modello continuista. Indicativa è in tale quadro la constatazione che, nei casi noti, il cambiamento del sistema politico è ricondotto dagli storici che lo hanno registrato all'egoismo individuale e spiegato nei termini di una decadenza dei costumi originari. Esemplare il caso di Teopompo, il quale identifica nell'egoismo individuale il principale fattore responsabile della crisi e della decadenza delle polieiai. ${ }^{73}$ Non dissimile lo schema adottato da Senofonte, il quale nella Ciropedia come nella Costituzione degli Spartani approda alla stessa conclusione: che nel sistema politico persiano, come in quello spartano, fosse

69 Per l'idea che Senofonte generalmente eviti di rievocare eventi della storia passata dei Greci nel quadro di ampie sezioni narrative: Rood 2012, 92-94. Sul disinteresse di Senofonte per gli agenti storici del cambiamento politico: Cawkwell 1979, 45; Breitenbach 1950, 147; Riedinger 1991, 252. Una prospettiva revisionista è quella di Buxton $(2017 ; 2018)$ che riconosce nelle Elleniche le tracce della rilevanza assegnata da Senofonte ai conflitti sociali (staseis), anche se non in diretto rapporto al rivolgimento costituzionale.

70 Sulla prospettiva continuista di Senofonte nell'Anabasi: Grethlein 2013b, 53-91.

71 Atack 2018, 171-176, in particolare 176: "Tensions between old and new aspects of constitutions, and manipulation of those histories of change, are a continuing feature of politeia writing seen in both Xenophon and Isocrates. This is not limited to discussing Athens, with its history of political change, but also appears in their writing on the Spartan politeia and on Persia and Egypt as counter-examples for Athens". Cf. anche Bordes $1982,225,352-353,441-442$.

72 Bordes 1982, 441; Cuniberti 2000; David 2007, 119-127; Fornis 2011, 222; Lupi 2017, 30-32.

73 Liddel 2010; Loddo 2016. 
responsabile della decadenza il mutamento dei costumi in direzione dell'egoismo individuale. ${ }^{74} \mathrm{Nel}$ penultimo capitolo (14) della Costituzione degli Spartani Senofonte afferma chiaramente che le leggi di Licurgo erano state alterate dalla condotta di singoli governanti. E la stessa affermazione di Senofonte - nel capitolo successivo e conclusivo (15) - che solo la carica regia aveva mantenuto le prerogative originarie, mentre le altre istituzioni avevano subito un processo di cambiamento "ancora in corso" al tempo in cui Senofonte scriveva il suo opuscolo, ${ }^{75}$ è direttamente collegata a quanto detto poco prima sul fattore che ha determinato la crisi della politeia: 1'egoismo individuale. ${ }^{76} \mathrm{E}$ uno schema interpretativo quello della decadenza del sistema politico delle origini indotto dall'egoismo individuale che Aristotele diversamente da Platone (cf. $\S 4)$ - sceglierà di superare, trovando nei rapporti di forza socio-economici i fattori scatenanti della metabole, ammettendo la possibilità del cambiamento (metabole) che migliora il sistema e non è in sé negativo, e ricostruendo lo sviluppo delle istituzioni in prospettiva storica e diacronica. Il caso della politeia spartana è -come vedremo-paradigmatico, considerato che Aristotele rifiuta la posizione che attribuiva a Licurgo l'organizzazione delle istituzioni dei Lacedemoni nella loro totalità ${ }^{77}$ invece che valorizzare la propettiva di Senofonte della degenerazione morale individuale come fattore che induce il cambiamento. ${ }^{78}$

Passando all'ambito della storiografia locale, il dato che conta enfatizzare nella prospettiva di una ricognizione che, come la nostra, ha come focus la politeia, è il rapporto cronologico che passa tra gli storici locali che si sono occupati di politeia e la grande storiografia.

La storiografia locale conosce un impulso notevole sul piano dei contenuti e della metodologia dopo la fine delle esperienze egemoniche raccontate dalla grande storiografia, dunque a partire dal tardo $\mathrm{V}$ secolo. ${ }^{79} \mathrm{Va}$ detto con chiarezza che collocare questa ricca e variegata produzione storiografica nel suo contesto cronologico non significa voler ridimensionare la tradizione della storiografia locale precedente ${ }^{80}$ un

\footnotetext{
74 Canfora 1992, 16-17.

75 X. Lac. 15.1. Devo al revisore anonimo, che ringrazio, il suggerimento di riflettere su questo passo.

76 Secondo Canfora 1992, 16-17, tale schema interpretativo è comune alla diagnosi tucididea per l'Atene postpericlea.

77 Lupi 2012; Polito 2017, 24-27.

78 Mentra il modello senofonteo è presente nella ricostruzione che offre Aristotele di alcuni personaggi centrali della storia spartana come Cinadone (HG 3.3-4) e Lisandro (HG 2.4.29, 2.4.7-9): cf. Paradiso 1994, 165-167, che, anche in quel caso, sottolinea la profonda originalità della rielaborazione aristotelica (170).

79 Porciani 2001a-b; Camassa 2010; Tober 2010; Thomas 2014b, 258.

${ }_{80}$ Per le origini della storiografia locale, il quadro resta incerto: Schepens 2001, 9; Clarke 2008, 193; Thomas 2014a, 149. Gli storici discutono sul medium utilizzato per la "polis history" delle origini, che alcuni ritengono fosse prevalentemente orale (Porciani 2001a, 13-27, 65-85). Un dato che non modifica il poco che conosciamo sul piano dei contenuti e soprattutto l'idea di storia che ne era alla base (Grethlein 2014, 235). È certamente notevole che uno dei maggiori specialisti di storiografia locale, Rosalind Thomas, riconosca l'interesse per le politeiai come precoce e politicamente rilevante in quanto funzionale a fissare l'identità di una polis in modo stabile (Thomas 2014a, 167; 2014b, 240). Anche Fowler $(2001,113)$ riconosce un interesse per la politeia come tratto distintivo della "local historiography" fin dalle sue origini. Per la fase delle origini non si parla di opere in prosa e le prime tracce di storia locale sono là dove i poeti arcaici dipendono da tradizioni storiografiche sulle fondazioni di città. Se si guarda alla letteratura in versi (Lasserre 1976; Orsi 1994; Bowie 2001; Grethlein 2014b, 238-241) un primo esempio è -nell'opera di Tirteo- la concezione di una Eunomia (Lasserre 1976, 126127; Orsi 1994, 154-156; Porciani 2001a; 2001b; 2006; Bowie 2001, 46) che precorre l'interesse degli storici più tardi per lo stile di vita e la politeia di Sparta (Tober 2010, 413-414). Guardando alle opere in prosa propriamente rivolte alla storia delle poleis, la fase delle origini è nota attraverso scarsissimi frammenti, spesso si tratta dei soli titoli di opere scomparse dai quali si ricava un interesse per le fasi fondative delle città (cosiddette ktiseis) attestate nei miti e nelle leggende locali (Orsi 1994, 149-152; Porciani 2006, 179-184).
} 
punto sul quale gli storici hanno criticato la posizione netta espressa da Felix Jacoby, il quale considerava la storiografia locale solo come una ramificazione secondaria della storiografia panellenica- ${ }^{81}$ La valorizzazione di questo rapporto cronologico serve piuttosto a valutare i caratteri distintivi della produzione del tardo $\mathrm{V}$ secolo (contenuti, metodo, finalità e modelli storiografici) nel quadro storico che li ha resi possibili.

Più significativo e meglio noto il caso ateniese. Le storie di Atene (Atthides) composte a partire dalla fine del V secolo e lungo i due secoli successivi, sebbene molto diverse fra loro, ${ }^{82}$ in quanto non tutte danno lo stesso rilievo al tema della politeia ${ }^{83}$ condividono però l'interesse per il passato della polis e per le sue istituzioni più rappresentative: un interesse che non è attestato prima delle crisi istituzionali ateniesi del $411^{84} \mathrm{e}$ del $404 .{ }^{85}$ Quelle storie locali di Atene sono il prodotto di quel clima politico. L'interesse per il tema delle origini costituzionali si spiega nel quadro di un dibattito politico che ha orientato gli autori delle storie locali di Atene e del quale non si trova traccia prima del $411 .^{86} \mathrm{E}$ un punto che Giorgio Camassa in uno studio sulle origini dell'attidografia ha messo chiaramente in luce. ${ }^{87}$ In questo quadro è notevole l'assenza di ogni evidenza di storie locali di Atene prima delle crisi oligarchiche del $411 \mathrm{e}$ del 404. È rimasta infatti un'ipotesi isolata quella argomentata da Santo Mazzarino, che una storia locale di Atene (Atthis) fosse stata composta prima che Erodoto componesse le sue Storie e che oggetto di questa narrazione fosse proprio la politeia delle origini. Al cuore dell'argomentazione di Mazzarino era l'idea che il dibattito sviluppatosi ad Atene dopo il 413 sulla costituzione ancestrale (patrios politeia) e attestato nei frammenti del trattato Perì politeías di Trasimaco (411 a.C.) dovesse contare su una fonte più antica e che fosse la politeia delle origini l'oggetto di quella anonima Atthis. ${ }^{88}$ Ripresa da Giovanni Maddoli, tale ipotesi non ha avuto altro seguito. ${ }^{89} \mathrm{Il}$ dato certo è che la più antica attestazione di un dibattito avente ad oggetto il carattere della patrios politeia nel V secolo (i frammenti di Trasimaco appunto) rappresenta l'unico valido terminus post quem per l'evidenza di una

81 Jacoby $(1949,147)$ osservava a proposito della storiografia locale che fosse "on the whole independent of, though connected with 'great historiography"' e che gli storici locali fossero animati dalla volonta di correggere o integrare la grande storiografia. Cf. ora Jacoby 2015, 32: "Greek historiography in the true sense did not, as one might believe, grow up out of a compilation of local histories. It has nothing whatever to do with these histories: in fact, horography is even later in time than historiography. Both genres of historical literature exist side by side and touch one another only so far as from time to time the historian uses the $\tilde{\omega} \rho 01$ [chronicles] as a source for details and, on the other hand, the horographer takes the kotvaì iotopíar [general histories] into account in the interest of local patriotism, that is, usually polemically". Il dibattito sviluppatosi sulla posizione di Jacoby può essere seguito attraverso l'ottima ricostruzione che ne offrono Porciani 2001b, 287-297; 2006, 174-182; Clarke 2008, 193-208, 230-243; Camassa 2010, 29-35; Thomas 2014a, 147-150. Sui rapporti, anche cronologici, della storiografia locale con la grande storiografia del V secolo: Schepens 2001, 15-17; Harding 2007, 187; Bravo 2007, 519; Clarke 2008, 193-208, 230-243.

82 Utili riflessioni in tal senso negli studi di Rhodes 1990, 73-81, e Harding 2007; 2008.

83 Sul punto, Jacoby 1949; Rhodes 1990; Clarke 2008, 180-193; Harding 2008; Bearzot - Landucci 2010.

84 Per l'idea che il 413 rappresenti un valido terminus post quem per l'evidenza di una discussione rivolta al tema della politeia delle origini, Rhodes 2006; Poddighe 2014, 129, 135. Cf. anche Bordes 1982, 162, 441.

85 Sulla propaganda politica che ha accompagnato le due rivoluzioni oligarchiche e le collegate questioni storiografiche: Sancho Rocher 2004; 2007; Shear 2011. Sempre utili i classici Fuks 1953; Finley 1971; Walters 1976; Mossé 1979.

86 Camassa 2010, 40-41.

87 Camassa 2010, 40-50. La misura del debito delle ricostruzioni storiche proposte dagli attidografi rispetto al dibattito contemporaneo è discusso: Rhodes 1990; Harding 1994; 2007; 2008; Bearzot 2010.

88 Mazzarino 1966, I 97-98, 559-560.

89 Maddoli 1985; diversamente: Porciani 2001a, 29-31. 
riflessione rivolta alle origini della politeia di Atene. Il "ritardo" con il quale è maturata una riflessione di tipo teorico sulla costituzione delle origini ${ }^{90}$ è del resto confermato dal fatto che anche la Politeia degli Ateniesi dello Pseudo-Senofonte non abbia cercato nel passato costituzionale di Atene modelli alternativi di democrazia. ${ }^{91}$ Un dato che appare sintomatico anche in mancanza di una precisa datazione dell'opera. ${ }^{92}$

Perciò è giusto spiegare la nascita stessa del genere attidografico, con l'esaltazione del passato locale che lo caratterizza, come la conseguenza di un clima politico propizio alla strumentalizzazione della storia antica di Atene..$^{93}$ In questo quadro si spiega l'interesse per la storia di istituzioni politiche rappresentative della politeia e perciò indagate a partire dalla fine del V secolo: nel caso ateniese, ci torneremo, è l'interesse per l'Areopago che, nell'opera del primo attidografo Ellanico di Lesbo ${ }^{94}$ e poi, nel corso dei secoli IV e III, nell'opera di Androzione e di Filocoro, ${ }^{95}$ appare trattato con esiti analoghi. ${ }^{96}$ Ugualmente significativo, pur nel quadro di opportune distinzioni tra gli Attidografi, il dato che proprio nel clima politico del tardo V secolo sia stato elaborato un modello di Atthis paradigmatico per contenuti, metodologia e finalità: quello di Ellanico. ${ }^{97} \mathrm{Al}$ centro della sua ricerca era il passato mitico e leggendario della polis ricostruito secondo un orizzonte di attesa ideologicamente e politicamente espressione dell'Atene della fine del V secolo. ${ }^{98}$ Le sue ricerche di carattere mitografico e cronografico e la ricostruzione proposta riguardo ai primi abitatori dell'Attica nella sua Attike syngraphe ${ }^{99}$ sono da valutare in diretto rapporto col clima politico che accompagna la fine della guerra del Peloponneso, quando la costruzione dell'identità ateniese si definisce soprattutto in rapporto al mito della politeia immutabile di Sparta e perciò cerca nelle origini ateniesi le tracce di un'altrettanto forte continuità culturale e politica. ${ }^{100}$

A quello stesso periodo storico si deve l'origine della ricerca antiquaria con quella che Momigliano ha chiamato una "rivoluzione nel metodo storico". ${ }^{101}$ È dalla congiunzione della sensibilità antiquaria con gli sviluppi dati alla crisi politica ateniese che sorgono gli interrogativi sul tempo remoto di Atene. ${ }^{102}$ Gli storici locali danno la propria ricostruzione su un dato problema della storia passata, spesso in

90 Interessanti le riflessioni svolte al riguardo da Giangiulio 2016.

91 Cf. Bordes 1982, 162 (citata alla nota 42).

92 Su questo disinteresse: Bordes 1982, 162, 441; Lenfant 2018. Per una discussione recente della questione relativa all'identità dell'autore, Gargiulo 2018.

93 Camassa 2010, 40-50. Posso solo rimandare alla trattazione di Thomas (2019, 316-357) sugli Attidografi che purtroppo è stata pubblicata dopo la stesura di questo articolo e non ho potuto utilizzare.

94 Su Ellanico: Jacoby 1949; Ambaglio 1980; Rhodes 1990, 76; Orsi 1994, 164; Möller 2001; Bravo 2007, 518; Ottone 2010; Camassa 2010, 40-41; Thomas 2014a, 165-166.

95 Sull'opera degli attidografi: Jacoby 1949; Harding 1977; 1994; 2007; 2008; Bearzot - Landucci 2010.

96 Harding 2007, 185; Tober 2010, 431.

97 Harding 2008, 3.

98 Ambaglio 1980, 9-192; Rhodes 1990, 76; Orsi 1994, 164-169; Möller 2001; Gehrke 2001; Bravo 2007, 518; Harding 2008, 3-4; Ottone 2010; Camassa 2010, 40-41; Thomas 2014a, 165-166.

99 Th. 1.97.2.

100 Per una puntuale descrizione dell'ambiente ateniese in cui si sviluppò la produzione di politeai di Sparta, Lupi 2017, 28-32. Per una messa a fuoco del rapporto tra continuum ideologico e dibattito sulla patrios politeia, Grethlein 2011, 261-262.

101 Momigliano 1984, 4.

102 Camassa 2010, 40. Per l'idea che le Atthides e le Politeiai di Sparta fossero soprattutto il prodotto di quelle esperienze politiche e della ricerca antiquaria: Bravo 2007; Harding 2007; Tober 2010, 431. Più scettici: Schepens 2001, 101; Clarke 2008, 180, 250. Sulle origini della ricerca antiquaria e la sua specificità sul piano della metodologia e degli interessi: Momigliano 1984, 4-8; 1991, 54-79; Bravo 2007. 
rapporto al dibattito politico contemporaneo, usando una ricca varietà di fonti e vagliando tradizioni diverse su questioni controverse. ${ }^{103}$ L'aspetto metodologico è essenziale per creare e rafforzare l'identità e il senso di appartenenza a una comunità: a questo scopo la ricerca antiquaria divenne una vera e propria "necessità". 104

Va osservato che lo spazio occupato dal tema della politeia nell'attidografia varia considerevolmente. Se si guarda all'opera degli "epigoni” di Ellanico, ovvero alle storie locali composte tra IV e III secolo da Clidemo, Androzione, Fanodemo e Filocoro, la varietà degli interessi appare evidente. ${ }^{105}$ L'interesse degli Attidografi era rivolto a questioni etimologiche come a problemi topografici, a questioni religiose come giuridiche, alla storia di tradizioni familiari come ad aspetti cultuali. Alla base della loro ricerca però appare la stessa esigenza di spiegare la fase delle origini della polis trattando aspetti fondamentali per l'identità della città. I temi che erano oggetto della loro indagine, anche quando sconfinavano nel racconto mitico, facevano parte della "memoria comune" degli Ateniesi ed erano perciò parte integrante di quella che Gehrke ha definito "social knowledge of the past". ${ }^{106}$ Il modello di "civic historiography" che quelle storie locali definiscono è quello di uno storico che mette al servizio del dibattito contemporaneo i risultati delle sue ricerche sul passato della polis. ${ }^{107}$ Non è necessario essere originari della città della quale si scrive la storia. Lo prova il caso citato di Ellanico di Lesbo: uno straniero, proveniente dal mondo degli alleati soggetti, capace però di intercettare le attese del pubblico di Atene. ${ }^{108}$ In questo quadro, la funzione assegnata alle ricerche su aspetti collegati alla storia della singola polis (e alla sua politeia) appare chiaro: si costruisce attraverso la politeia un passato mitico nel quale collocare i modelli politici di volta in volta elaborati come i più funzionali a spiegare il presente e a legittimarlo. ${ }^{109}$

La ricerca dello storico locale dà risposte in linea con l'esigenza della comunità politica di fissare la sua identità in modo stabile. ${ }^{110}$ Il fine della sua ricerca storica appare in definitiva quello di cercare nel passato ciò che legittima il presente nel quadro di quella che gli storici definiscono "intentional" o "designer" history. ${ }^{111}$

Ad accomunare questa variegata produzione è la funzione che si dà allo studio della storia della polis, delle origini delle sue istituzioni più rappresentative. La comparazione fra $\mathrm{i}$ diversi generi di storiografia locale testimonia questa identifica

103 Schepens 2001, 24. Su metodo, Huxley 1973; Momigliano 1984, 4-8; 1991, 54-79; Bravo 2007.

104 Nicolai 2007, 17.

105 Jacoby 1949; Rhodes 1990; Harding 2007; 2008; Bearzot - Landucci 2010.

106 Gehrke 2001, 286; Harding 2007, 183.

107 Boffo 1988, 34-35, 37-38; Schepens 2006; Clarke 2008, 183; Thomas 2014b, 242.

108 Harding 2008, 3-4; Camassa 2010, 40. Sui cosiddetti “travelling historians": Schepens 2006; Thomas 2014b, 242.

109 Murray 1993b; Boedecker - Raaflaub 1998; Foxhall - Gehrke - Luraghi 2011. Cf. Harding 2007, 183: "For the authors of local histories these 'mythical' accounts were embedded in oral tradition, which for their community was its 'history'. E ancora: "This type of mythico-historical tradition, which represents the way a 'society interprets and understands itself' through its 'social knowledge of the past', has been given the more innocuous title "intentional history"'. Cf. anche Gehrke 2001, 286. Sul valore sociale (al servizio della intentional history) che viene riconosciuto unanimamente all'opera degli Attidografi: Harding 2007, 183, 187; Bravo 2007, 517; Thomas 2014b, 240.

110 Harding 2007, 187, sulla finalità della polis di "establish its identity through its origins, cults, and traditions, specifically in regard to its right to its territory". Clarke 2008, 290-291, 311-319; Thomas 2014b, 241. Cf. ora Thomas $2019(3,15,152)$ sulla rappresentazione, nell'ambito della storiografia locale, della storia delle poleis come un continuum dalla fase delle origini, ad esempio nei casi della storia di Nasso (150) e Megara (169).

111 Gehrke 2001, 286; Nafissi 2009, 118. Cf. ora Canevaro 2017; Barbato 2017. 
finalità: quando gli aspetti peculiari dell'attidografia sono messi a confronto -come ha fatto Roberto Nicolai- con i generi contigui delle politeiai e con la produzione antiquaria, appare chiaro che la finalità eziologico-documentaria e la sensibilità antiquaria degli storici locali fossero giustificate non come sterile esercizio erudito ma nell'ambito della volontà di rafforzare l'identità locale ateniese facendo leva sulla memoria del passato e rivestendo così un ruolo ideologico. ${ }^{112}$

Il dato che nella nostra prospettiva appare più significativo, nel confronto con la grande storiografia, è il ricorso da parte degli storici locali allo stesso modello di rappresentazione storiografica: mentre cambiano i contenuti e la metodologia -la storiografia locale infatti sceglie temi omogenei ed evita le ampie sequenze narrative proprie del racconto monografico delle egemonie del V secolo- ${ }^{113}$ il modello storiografico utilizzato è quello della "static historiography" o "continuous history".

È un aspetto che gli specialisti della storiografia locale riconoscono nettamente: la funzione assegnata alla politeia è rappresentare la storia della polis nel segno della continuità col passato. ${ }^{114}$ Scrivere la storia di una polis significa affermare la sua peculiare identità davanti al resto del mondo greco ${ }^{115}$ rivolgendosi sia all'esterno che all'interno della comunità, ${ }^{116}$ senza soluzione di continuità rispetto alla fase delle origini e pertanto, come scrive Rosalynd Thomas "cristallizing a particulare vision of that polis". ${ }^{117}$

A dimostrare questo carattere sono i casi meglio noti di Sparta e Atene.

Gli studi condotti sulla storia di Sparta nel quadro della storiografia locale mostrano non solo che la storia delle origini di Sparta è stata raccontata dagli storici antichi a partire dalla origini della sua politeia,${ }^{118}$ ma che nelle storie locali di Sparta, la politeia è lo strumento esclusivo di una rappresentazione storica che nella fase delle origini cerca l'atto fondativo di un ordine politico la cui acquisizione è motivo di orgoglio della polis presente. ${ }^{119}$

La "misura" in cui gli storici spartani hanno contribuito all'elaborazione di quel modello storiografico resta discussa. Daniel Tober crede di poter riconoscere la presenza di una storiografia locale spartana che si esprimeva nella scrittura della politeia licurgica e si deve ad autori del IV secolo quali Tibrone, Pausania (II) e Lisandro, i quali, secondo testimoni antichi, tra gli altri Aristotele, avrebbero scritto dei libelli su questioni inerenti la politeia spartana. ${ }^{120}$ Diversamente, Marcello Lupi

112 Nicolai 2010. Sulla specificità di politeiai e Atthides: Bravo 2007, 521; Harding 1977, 148-160, 149, 158; 2007, 183-185; Clarke 2008, 180-183, 316; Thomas 2014a, 167. Sulla rilevanza politica della storiografia locale: Orsi 1994, 164-169; Schepens 2001, 7-24; Clarke 2008, 175-193, 316; Thomas 2014a; 2014b; Tober 2017, 470 n. 54.

113 Momigliano 1984, 4-8; 1991, 54-79. Sbagliato però negare finalità politica alla storia locale: Schepens 2001, 13; 2006, 101; Bravo 2007, 516; Clarke 2008, 175-193.

114 Porciani 2001a; 2001b; Fowler 2001, 113; Harding 2007, 185; Clarke 2008, 276-277, 283-287, 290-291; Thomas 2014b.

115 Lasserre 1976, 134; Harding 2007, 183; Thomas 2014b, 241; Tober 2017, 461.

116 Cf. Tober 2017 sulla peculiarità delle forme comunicative e delle finalità rispetto ai due tipi di audiences.

117 Thomas 2014b, 240. Cf. anche Clarke 2008, 283-287, 290-291 e 315 "in a variety of groups, bonds within the group are reinforced by a sense of continuity that comes from a shared knowledge (or pretended knowledge) of key figures and incidents in its past".

118 Cf. Tober 2010, 428: "The Spartan formulation of early Spartan history was the politeia"; 425: "For a Spartan, to write the history of the city of Sparta was to write about the politeia of Lykourgos; conversely, to talk politics was to talk about the past". Cf. anche Millender 2001, 123-125; Nafissi 2009; Lupi 2012; 2017.

119 Restano tra le pagine più illuminanti quelle di Murray 1993b. Cf. anche Paradiso 1995; 2000; Luraghi 2001; 2008; Nafissi 2009; Tober 2010.

120 Discussione di fonti e studi precedenti su questa produzione storiografica in Tober 2010; Lupi 2010; 2012; 2017; Polito 2017, 24-25. 
riconduce lo sviluppo di politeiai spartane all'ambiente dei circoli oligarchici ateniesi che guardavano con ostilità alla democrazia ateniese del tardo V secolo, identificando nelle Politeiai di Crizia e Senofonte il modello della riflessione storiografica su Sparta ${ }^{121}$ e avanzando in questo quadro la tesi che Tibrone fosse uno pseudonimo di Senofonte. ${ }^{122} \mathrm{Si}$ tratta di materiale scarsamente attestato, va detto, ma quel poco che gli studi hanno messo a fuoco riguardo ai contenuti di quelle storie locali è, nella prospettiva della nostra riflessione, piuttosto significativo, anche dal punto di vista del rapporto con i modelli elaborati negli ambienti ateniesi. L'impostazione adottata in quelle storie locali appare infatti coincidente con quella della grande storiografia e si manifesta nella forma di una "static civic historiography" che, con le parole di Daniel Tober "did not move beyond the ktisis: the Lykourgan politeia". ${ }^{23}$

Nell'opera degli attidografi gli studiosi riconoscono caratteri analoghi. ${ }^{124}$ Anche nelle storie di Atene, le fasi più antiche della politeia $-\mathrm{o}$ delle sue istituzioni più rappresentative- appaiono senza soluzione di continuità col presente. ${ }^{125}$ Un esempio significativo si trova nelle rappresentazioni offerte a proposito dell'Areopago nella fase delle origini. Secondo Philip Harding (ma già secondo Felix Jacoby) quella ricostruzione rappresenta "the best example of the way local history interpreted the past in light of the present". ${ }^{126}$ Diversamente dalla ricostruzione offerta da Aristotele nell'Athenaion Politeia ${ }^{127}$ dove l'Areopago delle origini esercita funzioni politiche tra le quali era il controllo della politeia ${ }^{128}$ gli attidografi lo rappresentano come corte di giustizia, con competenze prevalenti (anche se non esclusive) in materia di delitti di sangue. ${ }^{129} \mathrm{Il}$ fatto che tale rappresentazione passasse per la "fiction" di vicende mitologiche collegate a processi per omicidio non muta il dato essenziale che gli attidografi proiettassero nel passato remoto della polis l'origine delle funzioni assegnate all'Areopago nel IV secolo. ${ }^{130}$

Provando a tirare una prima linea su quanto fin qui considerato, il dato notevole che manifesta il pensiero storico rivolto alla politeia, nella grande storiografia come nella storiografia locale, è una sorta di "resistenza" alla valorizzazione sul piano teorico dei processi storici di cambiamento. Le forme di questa resistenza sono affini e riconducibili a due modalità principali: da un lato, è la costruzione di una prospettiva continuista nella quale pensare il passato senza soluzione di continuità col presente e quale momento originario di tutte le realizzazioni prodotte nel corso del tempo dalle principali poleis (Atene e Sparta) in ambito costituzionale e legislativo; dall'altro, è il ridimensionamento (talvolta l'obliterazione) dei cambiamenti intervenuti nella politeia e del concetto stesso di cambiamento. Analoghe, nella

121 Lupi 2017, 29-30.

122 Lupi 2010.

123 Tober 2010, 431; 2017. Utili anche Jacoby 1949, 289 n. 113; Orsi 1994, 154-156; Millender 2001, 123-125; Thomas 2014a, 167.

124 Cf. Tober 2010, 431: "The Spartan model in which all aspects of the politeia are attributable to Lykourgos, in fact, may very well have influenced the Atthidographic conception of early Athenian history".

125 Per il carattere della continuità nella tradizione attidografica, Thomas 2019, 316-357.

126 Harding 1994, 61-91; 2007, 185-188; 2008, 205-209. Cf. anche Chambers 1973.

127 Arist. Ath. 3.6, 8.2.

128 Analisi del carattere della ricostruzione aristotelica, anche nel confronto con la tradizione attidografica, in Poddighe 2014, 154-167, 245-258, 331-343.

129 FGH 323a F1; 324 F3-4; 325 F30; 328 F20, F196. Harding 2007, 185; Wallace 1989, 3-47; Tober 2010, 431: "Atthidography in general gave the primal Areiopagos the same responsibilities as the fourth-century court".

130 Harding 2008, 205-209. 
grande storiografia e nella storiografia locale, appaiono l'assenza di un preciso interesse ad affrontare il problema "storico" dell' evoluzione costituzionale sul piano teorico, ovvero in una prospettiva universale, e la scelta di non collocare la storia della politeia entro segmenti cronologici ampi. Nella grande storiografia come nella storiografia locale, alla funzione sociale della cosiddetta civic historiography assolve la concezione di una politeia da sempre uguale a se stessa.

\section{La metabole politeion nella riflessione storiografica e nel pensiero politico prima di Aristotele}

Uno specifico aspetto della questione riguarda lo spazio che il tema del cambiamento costituzionale occupa -nella riflessione storiografica e nel pensiero politico- nella prospettiva della storia universale. Una recente messa a fuoco del problema si deve a Peter Liddel che in uno studio del 2010 "Metabole Politeion as Universal Historiography", ha voluto riconsiderare in modo critico, anzi sfidare, l'idea dominante che il tema del cambiamento della politeia fosse scarsamente affrontato dagli storici greci fino ad Aristotele. ${ }^{131}$ I risultati problematici cui questo tentativo approda sono tuttavia ammessi dallo stesso studioso, ${ }^{132}$ il quale finisce per constatare come nell'opera degli storici presi in considerazione, Erodoto e Tucidide, i processi di cambiamento dei regimi politici non appaiono valutati nell'ottica di una storia universale ovvero con lo scopo di rintracciare linee di sviluppo delle trasformazioni politiche delle poleis che fossero universalmente valide. Manca questo tipo di prospettiva nel celebre e cosiddetto logos tripolitikos narrato da Erodoto ${ }^{133}$ e che racconta la discussione tra i maggiorenti persiani su quale fosse la forma di governo migliore da dare ai Persiani tra quelle democratica, oligarchica o monarchica. Sebbene, infatti, quel logos introduca nel racconto storico elementi di teoria costituzionale, a mancare è la volontà di definire linee di sviluppo universalmente valide, quelle che Liddel definisce "universally applicable developmental patterns which might go some way in explaining the past or even predicting the future". ${ }^{134}$ Neppure quando Erodoto riflette sulla metabole che dalla tirannide dei Pisistratidi ha portato alla democrazia di Clistene, lo sviluppo del pensiero erodoteo è capace di sviluppare un'analisi universalmente valida (che non sia l'idea erodotea che ogni egemonia è soggetta al cambiamento ineluttabile). ${ }^{135}$ L'impostazione data al racconto erodoteo evita la formulazione di schemi di interpretazione dei processi di cambiamento che possano essere universalmente applicati. ${ }^{136}$ Mentre è evidente in Erodoto la consapevolezza che la metabole costituzionale seguita alla caduta della tirannide è stata determinata a partire dai mutati rapporti di forza tra i pochi e i molti,

131 Liddel $(2010,25)$ definisce il suo tentativo "a challenge to the current scholarly tendency to conceive of an amnesiac inclination among the Greeks states when it came to thinking about constitutional transformation".

132 Liddel 2010, 26: "This search to identify metabole politeion as a stratagem for thinking about universal historical change has admittedly proved problematic". Liddel riconosce nell'opera di Polibio il primo uso universalistico del tema della metabole. Così già Châtelet 1974, 439; Alonso-Núñez 1990, 188-189; 2002, 72-80.

133 Hdt. 3.80.

134 Liddel 2010, 16.

135 Hdt. 5.58-66. Cf. Canfora - Corcella 1992.

136 Liddel 2010, 16. Un altro limite della visione erodotea è, secondo Liddel $(2010,19)$ che "the shift of power to the hand of many represented progress" (Hdt. 5.78), ciò che si spiega nel quadro di una "linear, non-cyclical view of how constitutional transformation works, at least in Athens" (24-25). 
a mancare nell'opera erodotea è l'interesse a definire le leggi generali delle rivoluzioni sociali che determinano il cambiamento costituzionale. ${ }^{137}$ Né questo interesse è riconoscibile nell'opera tucididea. Neanche quando Tucidide riflette sulle leggi ineluttabili che conducono al declino delle comunità politiche il tema appare affrontato sul piano della storia universale. Anzi, come lo stesso Liddel riconosce chiaramente, le leggi ineluttabili che fondano la visione tucididea sono incompatibili con un'analisi che cerchi gli agenti storici del cambiamento nelle dinamiche socioeconomiche e nella contrapposizione tra i pochi e i molti. Si tratta di un carattere della storiografia tucididea che anche Andrew Lintott e Rosalind Thomas hanno riconosciuto, in particolare a partire dall'analisi delle rivoluzioni politiche (staseis) verificatesi nelle comunità di Corcira, Anfipoli ed Argo e per le quali Tucidide non offre una ricostruzione attenta agli agenti socio-economici di quei rivolgimenti né alle leggi generali che li regolano. ${ }^{138}$

Se nella riflessione degli storiografi appare ben riconosciuta l'assenza di un interesse per lo sviluppo teorico del tema del mutamento politico in una prospettiva universale, nella riflessione teorica che ha fatto del tema della metabole politeion un tema centrale di riflessione -quella di Platone- è ugualmente evidente la scelta di non applicare la riflessione teorica alla storia sociale delle poleis. Anche in questo caso l'analisi di Liddel mette bene a fuoco il problema. Lo studioso parte dalla constatazione che nella Repubblica "the theory of political transformation is absolutely central" ma considera, al tempo stesso, che quel modello teorico non si alimenta dello studio di casi storici e oscura il ruolo che nei rivolgimenti costituzionali assume la contrapposizione fra i gruppi sociali. Ad essere decisivi in quell'impianto teorico sono invece i comportamenti individuali dei governanti "the deviant behaviour of the ruling class, who fall victim to human passions, is the cause of these changes (546d)". A impedire lo sviluppo del tema della metabole costituzionale in una prospettiva di storia universale è in definitiva -come osserva Liddel- l'assenza di una congiunzione tra teoria e storia: "The Republica's analysis of political transformation was carried out without historical references". E anche quando, nelle Leggi, la riflessione è rivolta alle cause del decadimento delle costituzioni persiana e ateniese, la ragione di quelle trasformazioni è ricondotta ai comportamenti individuali invece che alla contrapposizione fra $\mathrm{i}$ gruppi sociali. ${ }^{139}$

Questo carattere della riflessione platonica sul tema della metabole, e più in generale il significato che assume la storia delle poleis nell'impianto teorico platonico, sono stati

137 Lintott 1982, 75-76; Thomas 2011. Liddel (2010, 16-17) colloca giustamente il caso erodoteo nella prospettiva di valutare "the significance for universalist thinking of ancient Greek perspectives on the shifting balance of power between individuals, large groups, and small groups" (16), riconosce che "the prospect of constitutional transformation (in terms of which groups participated in politics) was a preoccupation of Greek politicians, historians, and philosophers" e che "it amounts to a strand of universalism in Greek historical thought", ma al tempo stesso ammette l'incapacità degli storici greci di "develop theories of power shifts which are universal in application" (17).

138 Lintott 1982, 98-120; Thomas 2011, 232: "Yet socio-economic analysis is significantly absent in Thucydides"; 238: "there is little on social class, conflicts between different groups of citizens, differing political aims". Così anche Liddel 2010, 21: "analysis of political change is dominated by the law of decay", Tucidide infatti "is cynical about the sociological force of constitutional transformation" e "did not perceive stasis as happening according to any particular patterns: indeed, his definition of stasis says that the organization within which it emerges is transformed from the onset condition". In definitiva, dunque, "Thucydides appears to avoid conceiving of constitutional transformation, in his own times, as structured or universalist in any sense" e "do not subscribe absolutely to a universalist view of constitutional change" (21).

139 Pl. $L g .694 a-701 d$. Cf. Liddel 2010, 22. 
più recentemente esaminati da Cynthia Farrar. ${ }^{140}$ I punti sui quali si sofferma il denso articolo di Farrar sono due: la centralità assegnata nella Repubblica al problema teorico della metabole politeion e l'irrilevanza che assume, rispetto a quell'impianto teorico, la storia dei rivolgimenti costituzionali verificatisi nel mondo greco e ai quali Platone fa cenno nelle Leggi. È il tema della mancata interazione fra storia e teoria politica.

La prospettiva della riflessione platonica sul tema dell'instabilità della politeia è -con le parole di Farrar- una "ahistorical perspective". ${ }^{141}$ Lo scarso interesse di Platone a fare oggetto di studio teorico la storia dei regimi esistenti è soprattutto riconoscibile nelle Leggi dove Platone nega la definizione di politeiai a regimi come la democrazia, l'oligarchia e la tirannide che, in quanto espressione del predominio di una fazione (stasioteiai), sono regimi irrimediabilmente lontani dal modello della politeia ideale. ${ }^{142}$ Anche quando la riflessione platonica evoca, nel terzo e quarto libro delle Leggi, i rivolgimenti politici che alcune specifiche realtà politiche (la democrazia ateniese e la monarchia persiana) hanno conosciuto nella loro storia, il risultato cui approda la riflessione platonica è l'inutilità dei contenuti di quelle vicende storiche ai fini di una riflessione che affronti il problema teorico del rivolgimento della politeia. ${ }^{143}$ Sulla diffidenza di Platone verso le politeiai storiche non si può che convenire con Lucio Bertelli il quale ha recentemente osservato «la diffidenza verso le costituzioni reali è confermata da quella specie di storia costituzionale che Platone disegna nel III libro attraverso i modelli canonici -Sparta, Persia, Atene--, il cui risultato è che la sorte delle costituzioni reali è accomunata in una condanna generale in quanto espressioni non dell'interesse comune, ma dell'interesse di una parte, pertanto le costituzioni reali -salvo in parte Sparta- sono stasioteiai, fazioni, non politeiai. ${ }^{144}$ La conseguenza di tale diffidenza è che anche $i$ temi storicamente rilevanti e affrontati nelle Leggi-quali la formazione delle città di Argo e Messene, la storia della monarchia persiana da Ciro a Serse, le guerre grecopersiane- non sono messi in relazione con la riflessione teorica rivolta al tema del cambiamento costituzionale, perché confliggono con il modello armonico di politeia che Platone ha in mente. Non si può elaborare una teoria politica sulle stasioteiai. ${ }^{145}$

La posizione espressa da Platone è particolarmente significativa, perché nega valore teorico al modello storiografico continuista adottato dagli storici del V secolo con riguardo al tema della politeia. È un aspetto che Cynthia Farrar affronta in modo particolare per la storia della politeia ateniese e che legge come una critica diretta alla posizione di Tucidide. ${ }^{146}$

Il tema della divaricazione fra teoria politica e indagine storiografica nell'opera platonica emerge anche nello studio di Kathryn Morgan che, tuttavia, rivolge la sua riflessione a un altro e più specifico tema: la misura del condizionamento esercitato dal modello continuista (il tema della stabilità della storia) nel pensiero storico di

140 Farrar 2013, 32-56.

141 Farrar 2013, 32-33, 35-37, 43-56.

142 Pl. Lg. 832c2-5. Farrar 2013, 48, 52. Sul punto, Schofield 2006, 31-43; Mulhern 2015, 86-87; Bertelli 2017a, 166.

143 Cf. ad esempio Farrar 2013, 36-38 su Pl. Lg. 3. 693d-701d.

144 Pl. $L g$. 832c; cf. 712c; 714a. Bertelli 2017b, 80.

145 Farrar 2013, 48 e 52.

146 Farrar 2013, 34-36, 39-43. Ugualmente scettici Castoriadis 1991, 8; Poddighe 2014, 70-71. Diversamente, altri studiosi affermano che si debba riconoscere un uso "didattico" della storia nella scelta di confrontare i modelli storici (cf. Pownall 2004) -ora per alienazione ora per assimilazione (Schofield 2006, 81) - alle forme ideali di modelli politici cui è rivolta la riflessione platonica. 
Platone. ${ }^{147}$ Pur nel quadro di differenti prospettive di ricerca, il dato che emerge più sistematicamente negli studi è che nella riflessione rivolta da Platone al tema dei cambiamenti della politeia è mancata la volontà di congiungere la riflessione teorica all'indagine storiografica sulle politeiai esistenti.

\section{Il contributo di Aristotele: metabole politeion e storia della polis in una prospettiva universale}

È solo nello spazio della ricerca che Aristotele ha condotto sulle politeiai storiche che il tema dell'instabilità costituzionale viene posto e affrontato con lo scopo di ricostruire la storia sociale delle poleis. La storia della polis viene così, per la prima volta, ricostruita attraverso i processi di mutamento costituzionale subiti nel tempo e lungo un arco cronologico ampio. ${ }^{148}$

Nel corpus di politeiai redatte all'interno della scuola aristotelica la funzione svolta dal tema del cambiamento costituzionale (metabole politeion) è stata chiarita almeno per i casi meglio noti. ${ }^{149}$ La storia della politeia ateniese è descritta nella forma di una serie di rivolgimenti che dalla fine del VII secolo arriva fino alla fine del $\mathrm{V}^{150} \mathrm{e}$, sebbene si discuta del fatto che il metodo impiegato per ricostruire e descrivere la storia della politeia ateniese fosse adottato per l'intero corpus delle politeiai ${ }^{151}$ qualche elemento significativo al riguardo lo si può ricavare guardando al caso spartano.

Un primo segnale del fatto che Aristotele avesse messo in "crisi" il modello storiografico elaborato nel V secolo per descrivere la politeia di Sparta è il fatto che nella Politica Aristotele non identifichi una stasis prelicurgica e che invece, la eunomia appare lo stato originario a cui fece seguito la stasis per i conflitti sorti tra gli aristocratici. ${ }^{152}$ Illuminanti le considerazioni che Marcello Lupi ha svolto con riguardo a questo tema. Lupi osserva che le ricerche dello Stagirita, pur fondandosi "proprio su quegli storici che negavano la presenza di staseis e metabolai" lo conducevano però in una direzione differente "vale a dire verso l'immagine di una città il cui assetto istituzionale era il frutto di una vicenda storica che non si esauriva nella figura del suo mitico legislatore". ${ }^{153}$ A dimostrarlo, secondo Lupi, il fatto, ben noto, che Aristotele "è certamente la fonte di Plutarco in relazione alla "Grande

147 Morgan 2012, 227-252.

148 Chambers 1961; Contogiorgis 1978, 141-160; Bordes 1982, 436-454; Polansky 1991; Poddighe 2014, 106-150; Lintott 2018.

149 Rassegna e discussione degli studi precedenti in Poddighe 2014, 18-23, 106-150. Ottime le raccolte di studi a cura di Polito - Talamo 2010; 2012.

150 Dopo la fine del V secolo Aristotele non riconosce significative metabolai nella politeia ateniese: Poddighe 2018b.

151 Murray 1993a, 205; Toye 1999, 136; Poddighe 2014, 121; Rhodes 2018; Thomas 2019, 377-385. Per l'idea che "la successione di metabolai che marcano la sezione diacronico-narrativa dell'Athenaion Politeias" fosse comune anche ad altre politeiai: Erdas 2017, 54-57, 62-67. Così anche Polito 2017, 32: "Un impianto del genere, sulla base di frammenti ed estratti, si può ipotizzare anche per altre Politeiai (direi con ragionevole certezza per quelle di Spartani, Cirenei, Corinzi, Tenedi, Sami, Cumani e Reggini, e con una certa probabilità per altre ancora). Inoltre il fatto stesso che Aristotele concepisca la continuità della comunità nel suo sviluppo indurrebbe a formulare l'ipotesi che una Politeia non potesse essere priva di una sezione diacronica dedicata appunto al dipanarsi di tale sviluppo".

152 Arist. Pol. 1306b22-1307a 5. Paradiso 1994, 161-170; Fornis 2016, 61 n. 137, per la dipendenza di questo modello da Senofonte e Eforo. Cf. anche Bertelli 2004; David 2007, 125-127; Lupi 2012.

153 Lupi 2012, 71-72. 
Rhetra" licurgica e al cosiddetto "emendamento" introdotto dai re Polidoro e Teopompo, ed è anche colui che attribuisce l'introduzione dell'eforato allo stesso Teopompo, individuando così nel regno di questo sovrano, tradizionalmente associato alla prima guerra messenica, un momento di crisi istituzionale". ${ }^{154}$ A dimostrarlo sarebbe anche, secondo Lupi, la circostanza che negli Excerpta Politiarum di Eraclide Lembo - cioè fra gli estratti che la tradizione manoscritta ci ha restituito di un'opera Sulle Costituzioni in cui Eraclide epitomava il contenuto delle Politeiai aristoteliche- ${ }^{155}$ la sezione dedicata a Sparta iniziava con le parole "alcuni attribuiscono a Licurgo l'intera costituzione degli Spartani": un'espressione che "lascia presumere che la posizione di Aristotele fosse differente". ${ }^{156}$ Secondo quell'epitome peraltro anche la Politeia degli Spartani (come quella degli Ateniesi) si divideva in due parti: una prima di carattere storico che si soffermava su Licurgo e le trasformazioni successive dell'ordinamento spartano, e una seconda sui costumi della città. ${ }^{157}$

I casi delle politeiai di Atene e Sparta mostrano che Aristotele avesse fatto della politeia la categoria euristica essenziale della sua riflessione sulla storia della polis e della metabole politeion lo strumento attraverso il quale collocare la storia di una singola polis in una prospettiva universale.

Nelle politeiai aristoteliche la ricerca storica sulla polis appare strettamente congiunta alla teoria politica. ${ }^{158} \mathrm{Ed}$ è alla teoria politica che bisogna guardare per trovare la giustificazione del valore euristico del tema della metabole politeion per la ricostruzione della storia sociale delle poleis. ${ }^{159}$

Lo studio del tema del mutamento nell'opera politica di Aristotele dimostra che il mutamento della politeia identifica per Aristotele ogni mutamento nella società che sia suscettibile di condizionare le strutture sociali e politiche. La gamma dei fenomeni implicati è molto ampia e va dai semplici avvicendamenti delle classi di governo alle sedizioni civili, dalle trasformazioni di un'oligarchia in una democrazia (e viceversa) al colpo di stato, dalla rivoluzione interna al gruppo di governo al tirannicidio e via dicendo. La "rivoluzione" non va intesa in senso moderno, poiché nella concettualizzazione aristotelica, quale che sia la portata e la modalità della transformazione politica, essa non distrugge le istituzioni fondamentali e la struttura sociale della polis.

Alla ricostruzione della storia delle poleis sono applicati gli schemi essenziali della teoria politica aristotelica: la teoria delle forme di governo e la concezione politica incentrata sul concetto di giustizia. In linea generale, il rapporto tra questi due schemi dà ragione di ogni processo storico di cambiamento: da un lato, i passaggi da una forma di governo ad un'altra sono influenzati dalle conseguenze dell'inosservanza dei principi di giustizia; dall'altro, sono i contrapposti principi di giustizia a informare e vitalizzare i progetti rivoluzionari di oligarchici e democratici, i due "partiti" che mirano a scalzarsi vicendevolmente e sulle cui vicende Aristotele si è soffermato con maggiore attenzione.

154 Lupi 2012, 71-72.

155 Polito 2001.

156 Lupi 2012, 72.

157 Lupi 2012, 71-72; 2017, 30-32. Cf. ora le condivisibili osservazioni svolte al riguardo da Polito 2017, 24-32.

158 Cf. ora Erdas 2017, 54-57, 62-67; Polito 2017, 11-43; Thomas 2019, 363-365.

159 Polito 2017, 11-43; Thomas 2019, 358-385. Cf. anche Dietze - Mager 2017, che dedica un'ampia sezione al contenuto delle politeiai in relazione alla storiografia locale (56-63). 
Grande spazio assume nella teoria aristotelica applicata alla storia delle poleis l'analisi del contenuto di questi due principi di giustizia e il loro significato per la lotta per il potere dei due partiti. Dai casi storici Aristotele attinge il materiale che consente di riconoscere e definire le leggi universali del cambiamento: ad esempio i rapporti di forza che possono fungere da catalizzatori del mutamento (per esempio il numero schiacciante dei democratici) così come le determinanti dirette di situazioni conflittuali che, secondo Aristotele, portano a trasformazioni politiche (l'eccesso nell'esercizio del potere, l'incuria o la negligenza di chi governa e via dicendo).

A determinare le differenze tra i modelli o le fasi costituzionali non sono, nella riflessione politica di Aristotele, fattori come la natura o la necessità, i quali infatti non spiegano né l'evoluzione storica di una costituzione né la diversità tra le costituzioni, ciò che conferma il valore euristico della metabole come paradigma interpretativo della storia politica e sociale. ${ }^{160}$ A determinare le differenze tra $\mathrm{i}$ modelli e le fasi costituzionali è invece, a giudizio di Aristotele, il cosiddetto dikaion, la nozione di giustizia, ovvero l'idea di interesse comune che rappresenta il fine della comunità politica. ${ }^{161}$ E a partire da questa considerazione che si possono comprendere $\mathrm{i}$ tratti distintivi dell'idea aristotelica della metabole. In particolare, il fatto che per Aristotele la metabole non identifica solo i cambiamenti totali di un ordine politico o sociale, ma anche il cambiamento limitato, purché capace di rivelare il nuovo eidos della giustizia. ${ }^{162}$

Dai casi storici Aristotele attinge il materiale che consente di riconoscere e definire le leggi universali del cambiamento. Sebbene non sviluppate e incorporate nella forma di una teoria generale della successione storica delle comunità politiche, ci sono delle leggi generali che regolano la storia della poleis e dunque delle politeiai. Innanzitutto, lo sviluppo delle tecniche di produzione (che hanno determinato la crescita delle città) e quindi la creazione di nuove forze sociali. ${ }^{163}$ Occorre valutare la nuova prospettiva adottata da Aristotele a partire dalla constatazione che nel IV secolo si erano costituiti "differenti campi di interesse materiale e scientifico" che avevano "una dimensione totalmente storica". ${ }^{64}$ La storia del susseguirsi di costituzioni che Aristotele ricostruisce in rapporto ai presupposti sociologici di quel processo di trasformazioni -ad esempio, attraverso il collegamento tra demografia e storia costituzionale- è il prodotto più tipico di questa ragione storica. ${ }^{165}$ Per Aristotele, che era uno "scienziato della politica", era necessario studiare la politeia secondo criteri scientifici. Il fine era quello di riconoscere la forma di ogni politeia e a questo fine diveniva indispensabile portare su un piano teorico e formale il fenomeno della trasformazione costituzionale. In questa direzione lo spingeva anche la cultura politica del suo tempo, impegnata da oltre mezzo secolo a discutere della forma della democrazia ateniese delle origini: una discussione rispetto alla quale Aristotele ha voluto porsi in modo critico. Non si trattava soltanto di studiare la politeia secondo criteri scientifici. Aristotele ha inteso fare ex novo la storia delle politeiai, partendo dalle praxeis -che sono l'oggetto della historia- ${ }^{166}$ e correggendo l'impostazione tradizionale degli storici i quali, a giudizio di Aristotele, si limitavano

\footnotetext{
160 Poddighe 2014, 39-41, 65-66.

161 Poddighe 2014, 14-17, 25-30, 41-54, 60-66, 106-107, 151; 2016.

162 Polansky 1991, 325, 332.

163 Contogiorgis 1978, 141-160; Lintott 2018.

164 Meier 1988, 366.

165 Poddighe 2014, 111-113.

166 Arist. Rh. 1360a 36-38.
} 
a dire il "cosa" e i "particolari" senza valutare (theorein) i genomena e le praxeis pertinenti un dato tema (quello costituzionale). Nella prospettiva di Aristotele, il problema da affrontare era lo svolgimento storico di un processo di mutamento del quale occorreva identificare, descrivere e spiegare gli sviluppi più significativi. Aristotele voleva identificare il cambiamento, capirne le origini e descriverlo, raccontarlo. Di qui l'interesse per alcuni indicatori in particolare, ad esempio per le leggi che rivelano, meglio di altri prodotti della politica, il cambiamento. ${ }^{167}$

È questo diverso interesse che spiega la distanza di Aristotele rispetto al modello narrativo degli storici del V secolo: la sua necessità di affrontare sul piano teorico il problema "storico" dell'instabilità costituzionale. ${ }^{168}$ Tra i caratteri distintivi dell'impostazione aristotelica è l'idea che lo svolgimento del processo storico di cambiamento della politeia vada compreso e descritto entro un quadro globale. La politeia rappresentava un tema capace di rivelare i nessi causali fra i genomena, di ordinarli secondo una sequenza intelligibile, e di offrire una "comprehensive view" sulla storia della polis, a patto però di iscriverla entro una visione sinottica e globale. ${ }^{169}$ L'idea che la storia della politeia debba essere studiata all'interno di una visione d'insieme è argomentata nel modo più esplicito nella Retorica. ${ }^{170}$ Il termine che Aristotele impiega nella Retorica per definire questa visione è synoran. ${ }^{171}$ Attestato solo a partire dal IV secolo, il verbo synoran ricorre di norma nell'opera aristotelica per definire la comprensione che passa per il ragionamento sinottico. Che si tratti di materie finanziarie o militari, di corpi legislativi o modelli costituzionali, per arrivare a conoscere occorre raggiungere una visione globale (synoran) alla quale si approda solo adottando il metodo comparatistico proprio della ricerca storica. ${ }^{172}$ E la sottolineatura dell'utilità del ragionamento per temi qualitativamente omogenei e razionalmente ordinati: un tipo di ragionamento capace di superare il limite dell'opera storica di cui si dice nella Poetica ovvero il fatto di cercare soltanto nel nesso posto dal tempo l'unità degli elementi particolari della realtà umana. Se infatti, come afferma Aristotele nel capitolo 23 della Poetica, il limite principale dell'opera storica è quello di adottare la dimensione temporale come unico nesso tra soggetti qualitativamente disomogenei, il rimedio è nella scelta di temi omogenei che rivelino per analogia e per differenza gli universali. ${ }^{173}$

All'interno di questo quadro teorico si definisce una nuova funzione civica dello storico: una funzione che passa, secondo Aristotele, per lo studio storico della politeia. La considerazione che lo studio storico delle politeiai serve allo storico della polis ad esprimere una diversa funzione sociale è da intendere nel senso che una delle finalità assegnate da Aristotele a quel tipo di ricerche è la possibilità di dare al dibattito politico contemporaneo contenuti storici verificati e "di qualità".

È il rovesciamento dell'idea che "non è utile" che la storia corregga il mito delle origini di una politeia. È al contrario l'affermazione dell'utilità sociale dello studio storico della politeia.

\footnotetext{
167 Bordes 1982, 441-454; Poddighe 2014, 46-53.

168 Canfora 1982, 389.

169 Poddighe 2019.

170 Poddighe 2017, 61-81.

171 Arist. Rh. 1357a 1-4; 1359b 19-32. Cf. Poddighe 2017.

172 Poddighe 2017, 69-75.

173 Poddighe 2017, 77.
} 
Che la sensibilità storico-antiquaria del Peripato fosse sollecitata da precisi intenti etici e politici, è stato giustamente osservato dagli storici. ${ }^{174}$ Per Aristotele la salvezza della politeia (la sua soteria) era soprattutto intesa come stabilizzazione degli assetti vigenti, con una correzione però che, nel caso aristotelico, è alimentata dallo studio di modelli politici del passato che possono rappresentare il paradigma per le opportune correzioni. Di qui il valore sociale e universale della ricerca storica sulla politeia che è studio dei casi reali e storici. Di qui l'idea aristotelica che il legislatore come il politico debbano studiare la storia delle costituzioni, delle leggi, delle guerre passate per conoscere la materia politica e gestire il presente, di qui l'idea che solo il "sapere storico" in materia di politeiai permette di agire terapeuticamente sul presente. La storia, come ha scritto Mauro Moggi "è in qualche misura l'alimento che nutre lo studioso della politica, il legislatore e l'uomo di stato e che mette questi ultimi in condizione di operare con cognizione di causa [...]. È chiaro tuttavia [...] che essa può svolgere un compito di questo genere soltanto se è in grado di offrire una serie di dati il più possibile autentici e rispondenti alla realtà, perché solo così i responsabili di una polis saranno in grado di suggerire le decisioni veramente più opportune e più utili da assumere di volta in volta su una serie di questioni che riguardano la sua stessa esistenza e il destino dei suoi politai".${ }^{175}$

\section{Bibliografia}

Alonso-Núñez, J. M.

(1990): "The Emergence of Universal Historiography from the 4th to the 2nd centuries B.C.”, [in] Verdin - Schepens - de Keyser (eds.), 1990, Leuven, 173-192.

(2002): The Idea of Universal History in Greece: from Herodotus to the Age of Augustus (=Amsterdam Classical Monographs 4), Amsterdam.

Ambaglio, D. (1980): L'opera storiografica di Ellanico di Lesbo (=Biblioteca di Studi Antichi 24. Ricerche di Storiografia Antica 2), Pisa.

Atack, C. (2018): "Politeia and the Past in Xenophon and Isocrates", [in] M. Tamiolaki (ed.), Xenophon and Isocrates. Political Affinities and Literary Interactions, Trends in Classics (=Special Issue 10), Berlin, 171-196 (http://dx.doi.org/10.1515/tc-2018-0009).

Ball, T. - Farr, J. - Hanson, R. L. (eds.), (1989): Political Innovation and Conceptual Change, Cambridge.

Barbato, S. (2017): "Using the Past to Shape the Future: Ancestors, Institutions and Ideology in Aeschin. 2. 74-78", [in] Franchi - Proietti (eds.), 2017, 213-254.

Bearzot, C. (2010): “L'orientamento politico degli attidografi: il caso di Androzione", [in] Bearzot - Landucci (a.c.), 2010, 113-128.

Bearzot, C. - Landucci, F. (a.c.), (2010): Storie di Atene, storia dei Greci: studi e ricerche di attidografia (=Contributi di storia antica 8), Milano.

Bearzot, C. - Canevaro, M. - Gargiulo, T. - Poddighe, E. (a.c.), (2018): Athenaion Politeiai tra storia, politica e sociologia: Aristotele e Ps-Senofonte, Atti del Convegno Internazionale di Studi (Cagliari 10-12 Maggio 2017), Milano.

Bertelli, L.

(1989): “Metabole politeion”, Filosofia politica 3/2, 275-326.

174 Cf. da ultimo Bertelli 2017a, 191; Camassa 2018, 100; Poddighe 2018a, 166.

175 Moggi 2017, 62. 
(2004): “La Sparta di Aristotele: un ambiguo paradigma o la crisi di un modello?", Rivista storica dell'antichità 34, 9-71.

(2017a): Politeia en logois. Studi sul pensiero politico greco, Torino.

(2017b): "Scienza della politica e politeia in Aristotele", La Parola del Passato 71, 7596.

Boedecker, D. - Raaflaub, K. A. (eds.), (1998): Democracy, Empire and the Arts in FifthCentury Athens (=Center for Hellenic Studies. Colloquia 2), Cambridge.

Boffo, L. (1988): “Epigrafi di città greche: un'espressione di storiografia locale”, [in] Studi di storia e storiografia antiche: per Emilio Gabba, Pavia, 9-48.

Bordes, J. (1982): Politeia dans la pensée grecque jusq'à Aristote, Paris.

Bowie, E. (2001): “Ancestors of Historiography in Early Greek Elegiac and Iambic Poetry?" [in] N. Luraghi (ed.), The Historian's Craft in the Age of Herodotus, Oxford, 45-66.

Bravo, B. (2007): “Antiquarianism and History", [in] Marincola (ed.), 2007, 515-527 (http:// dx.doi.org/10.1002/9781405185110.ch53).

Breitenbach, H. R. (1950): Historiographische Anschauungsformen Xenophons, Freiburg.

Buxton, R. F.

(2017): "Modeling Hegemony through Stasis: Xenophon on Sparta at Thebes and Phlius", Illinois Classical Studies 42/1, 21-40 (https://doi.org/10.5406/illiclasstud.42.1.0021).

(2018): "Forging Unity, Exporting Unrest: Xenophon and Isocrates on Stasis", Trends in Classics 10/1, 154-170 (https://doi.org/10.1515/tc-2018-0008).

Camassa, G.

(2010): "L'attidografia nella storia degli studi”, [in] Bearzot - Landucci (a.c.), 2010, 29-51. (2018): "Riflessioni sul concetto di soteria in Aristotele, a partire dalla Politica", [in] Bearzot - Canevaro - Gargiulo - Poddighe (a.c.), 2018, 87-103.

Canevaro, M. (2017): “La memoria, gli oratori e il pubblico nell'Atene del IV secolo", [in] Franchi - Proietti (eds.), 2017, Trento, 171-212.

Canfora, L.

(1982): “Gli storici greci”, [in] L. Firpo (a.c.), Storia delle idee politiche, economiche e sociali, vol. I, Torino, 374-419.

(1988): Tucidide. L'oligarca imperfetto, Roma.

(1992): "Nota", [in] G. F. Gianotti (a.c.), Le tavole di Licurgo. Senofonte, Palermo.

(2011): "Il corpusculum degli Epitafi ateniesi", Quaderni di Storia 74, 5-24.

Canfora, L. (a.c.), (1996): Tucidide. La guerra del Peloponneso, Torino.

Canfora, L. - Corcella, A. (1992): "La letteratura politica e la storiografia", [in] G. Cambiano - L. Canfora - D. Lanza (a.c.), Lo spazio letterario della Grecia antica, vol. I. La produzione e la circolazione del testo. La polis, Roma, 437-454.

Castoriadis, C. (1991): Philosophy, Politics, Autonomy. Essays in Political Philosophy, New York.

Cawkwell, G. (1979): “Introduction”, [in] R. Warner (trans.), Xenophon: A History of My Times (Hellenica), Harmondsworth, 7-46.

Chambers, J. T. (1973): Studies on the fourth-century Athenians'view of their past, Ann Arbor.

Chambers, M. (1961): “Aristotle's Forms of Democracy", Transactions of the American Philological Association 92, 20-36 (http://dx.doi.org/10.2307/283799).

Châtelet, F. (1974): La nascita della storia. La formazione del pensiero storico in Grecia, Bari.

Clarke, K. (2008): Making Time for the Past. Local History and Polis, Oxford (http://dx.doi. org/10.1093/acprof:oso/9780199291083.001.0001).

Contogiorgis, G. D. (1978): La théorie des révolutions chez Aristote (=Bibliothèque de Philosophie du Droit 22), Paris. 
Cuniberti, G. (2000): “Lakedaimonion Politeia: priorità e originalità nel dibattito sulle politeiai-modello di Sparta e Creta”, Studi Italiani di Filologia Classica 18, 99-111.

D'Angour, A. (2011): The Greeks and the New. Novelty in Ancient Greek Imagination and Experience, Cambridge (http://dx.doi.org/10.1017/CBO9781139003599).

David, E. (2007): "Myth and Historiography: Likourgos", [in] G. Herman (ed.), Greeks between East and West. Essays in Greek Literature and History in Memory of David Asheri, Jerusalem, 115-135.

Dietze-Mager, G. (2017): "Die Politeiai des Aristoteles und ihre Beziehung zu den Nomima Barbarika", Mediterranea 2, 35-72 (http://dx.doi.org/10.21071/mijtk.v0i2.6715).

Dodds, E. R. (1973): The Ancient Concept of Progress and other Essays on Greek Literature and Belief, Oxford.

Edelstein, L. (1967): The Idea of Progress in Classical Antiquity, Baltimore.

Epstein, S. (2011): "Direct democracy and minority rule", [in] G. Herman (ed.), Stability and Crisis in the Athenian Democracy, Stuttgart, 87-102.

Erdas, D. (2017): "Frammenti sulle costituzioni, costituzioni di frammenti. Ipotesi per una struttura delle politeiai aristoteliche", La Parola del Passato 71, 45-73.

Farrar, C. (2013): "Putting History in its Place: Plato, Thucydides, and the Athenian Politeia", [in] V. Harte - M. Lane (eds.), Politeia in Greek and Roman Philosophy, Cambridge, 3256 (http://dx.doi.org/10.1017/CBO9781139096843.004).

Finley, M. I.

(1971): The ancestral constitution. An inaugural lecture, Cambridge.

(1986): "Revolution in antiquity", [in] R. Porter - M. Teich (eds.), Revolution in History, Cambridge, 47-60 (http://dx.doi.org/10.1017/CBO9781316256961.003).

Fornis, C.

(2011): "Ideas e imágenes de Esparta entre los griegos de época clásica", [in] J. M Cortés Copete - E. Muñiz Grijalvo - R. Gordillo Hervás (eds.), Grecia ante los imperios. V Reunión de historiadores del mundo griego (=SPAL Monografías 15), Sevilla, 217-228.

(2016): Esparta. La Historia, el Cosmos y la Leyenda de los Antiguos Espartanos, Sevilla.

Fouchard, A. (2000): "La citoyenneté athénienne vue par Péricles", [in] C. Fiévet (ed.), Invention et réinvention de la citoyenneté, Pau, 35-44.

Foxhall, L. - Gehrke, H. J. - Luraghi, N. (eds.), (2010): Intentional History. Spinning Time in Ancient Greece, Stuttgart.

Franchi, E. - Proietti, C. (eds.), (2017): Conflict in Communities. Forward-looking Memories in Classical Athens (=Università degli Studi di Trento. Dipartimento di Lettere e Filosofia. Quaderni 7), Trento.

Fuks A. (1953): The Ancestral Constitution. Four Studies in Athenian Party Politics at the End of the Fifth Century B.C., London.

Gargiulo, T. (2018): "Per un profilo dell'anonimo autore dell'Athenaion Politeia pseudosenofontea", [in] Bearzot - Canevaro - Gargiulo - Poddighe (a.c.), 2018, 291-308.

Gehrke, H. J.

(2001): "Myth, History, and Collective Identity: Uses of the Past in Ancient Greece and Beyond”, [in] Luraghi (ed.), 2001, 286-313.

(2011): "Myth, History, Politics, Ancient and Modern", [in] J. Marincola (ed.), Greek and Roman Historiography. Oxford Readings in Classical Studies, Oxford, 40-71.

Giangiulio, M.

(2001): "Constructing the Past: Colonial Traditions and the Writing of History. The Case of Cyrene”, [in] Luraghi (ed.), 2001, 116-137. 
(2016): “Le politeiai delle città della Magna Grecia: peculiarità e dinamiche”, [in] Poleis e politeiai nella Magna Grecia arcaica e classica, Atti del cinquantatreesimo Convegno di Studi sulla Magna Grecia, Taranto 26-29 settembre 2013, Taranto, 203-214.

Goldhill, S. - Osborne, R. (eds.), (2006): Rethinking Revolutions through Ancient Greece, Cambridge.

Goušchin, V. (2019): "Plutarch on Cimon, Athenian Expeditions, and Ephialtes' Reform (Plut. Cim. 14-17)", Greek, Roman and Byzantine Studies 59/1, 38-56.

\section{Grethlein, J.}

(2010): The Greeks and their Past. Poetry, Oratory and History in the Fifth-Century $B C E$, Cambridge.

(2011): "Historia magistra vitae in Herodotus and Thucydides? The exemplary use of the Past and ancient and modern temporalities", [in] Lianeri (ed.), 2011, 247 263.

(2013a): "Democracy, Oratory, and the Rise of Historiography", [in] J. P. Arnason - K. A. Raaflaub - P. Wagner (eds.), The Greek Polis and the Invention of Democracy. A Politicocultural Transformation and Its Interpretations, Malden, 126-143 (https://doi. org/10.1002/9781118561768.ch5).

(2013b): Experience and Teleology in Ancient Historiography. 'Futures Past' from Herodotus to Augustine, Cambridge (http://dx.doi.org/10.1017/CBO9781139628815).

(2014a): "The Value of the Past Challenged: Myth and Ancient History in the Attic Orators", [in] J. Ker - C. Pieper (eds.), Valuing the Past in the Greco-Roman World (=Mnemosyne Supplements 369), Leiden, 326-354 (http://dx.doi. org/10.1163/9789004274952_014).

(2014b): "The Many Faces of the Past in Archaic and Classical Greece", [in] K. A. Raaflaub (ed.), Thinking, Recording, and Writing History in the Ancient World, Malden, 234-255 (https://doi.org/10.1002/9781118412541.ch12).

Harding, $\mathrm{P}$.

(1977): "Atthis and Politeia", Historia 26/2, 148-160.

(1994): Androtion and the Atthis. The Fragments Translated with Introduction and Commentary, Oxford.

(2007): “Local History and Atthidography", [in] Marincola (ed.), 2007, 180-188 (https:// doi.org/10.1002/9781405185110.ch14).

(2008): The Story of Athens. The Fragments of the Local Chronicles of Attika, Edited and Translated and with an Introduction and Commentary, London (https://doi. org/10.4324/9780203448342).

Hodkinson, S. (2005): “The Imaginary Spartan Politeia”, [in] M. H. Hansen (ed.), The Imaginary Polis (=Acts of the Copenhagen Polis Centre 7; Historisk-filosofiske Meddelelser 91), Copenhagen, 222-281.

Hornblower, S. (1995): "The Fourth-Century and Hellenistic Reception of Thucydides", Journal of Hellenic Studies 115, 47-68 (https://doi.org/10.2307/631643).

Huxley, G. (1973): “Aristotle as Antiquary”, Greek, Roman and Byzantine Studies 14/3, 271-286. Jacoby, F.

(1949): Atthis. The local chronicles of ancient Athens, Oxford.

(2015): On the Development of Greek Historiography and the Plan for a New Collection of the Fragments of the Greek Historians (=Histos Supplements 3), Newcastle.

Kirkwood, G. M. (1972): “Thucydides' Judgment of the Constitution of the Five Thousands (VIII, 97, 2)", American Journal of Philology 93/1, 92-103 (https://doi. org/10.2307/292904). 
Lanza, D. (2006): “Percorsi dell'ateniesità, Ítaca”, Quaderns catalans de cultura clàsica 22, 23-32 (https://doi.org/10.2436/20.2501.01.3).

Lasserre, F. (1976): “L’Historiographie grecque à l'époque archaique”, Quaderni di Storia 4, 113-142.

Lenfant, D.

(2018): “Quel modèle pour l'oligarque ? Le passé, l'ailleurs et l'utopie dans la Constitution des Athéniens du Pseudo-Xénophon", [in] Bearzot - Canevaro - Gargiulo - Poddighe (a.c.), 2018, 309-322.

(c.s.): "The Funeral Oration as the Self-Portrait of Athenian Democracy", [in] D. Pritchard (ed.), The Athenian Funeral Oration, Cambridge.

Lianeri, A. (ed.), (2011): The Western Time of Ancient History: Historiographical Encounters with the Greek and Roman Pasts, Cambridge (http://dx.doi.org/10.1017/ CBO9780511975998).

Liddel, P. (2010): "Metabole Politeion as Universal Historiography”, [in] P. Liddel - A. Fear (eds.), Historiae Mundi. Studies in Universal Historiography, London, 15-29.

Lintott, A.

(1982): Violence, Civil Strife and Revolution in the Classical City 750-330 BC, London (https://doi.org/10.4324/9781315778662).

(2018): Aristotle's Political Philosophy in its Historical Context. A New Translation and Commentary on Politics Books 5 and 6, London (https://doi. org/10.4324/9780203703311).

Loddo, L. (2016): “Cambiamenti costituzionali nei Philippika di Teopompo di Chio", Incidenza dell'Antico 14/2, 175-206.

Loraux, N. (1996): "Clistene e i nuovi caratteri della lotta politica”, [in] Settis, S. (a.c.), I Greci. Storia, cultura, arte e società, 2.1, Torino, 1083-1110.

Lupi, M.

(2010): "Tibrone, Senofonte e le Lakedaimonion Politeiai del IV secolo (a proposito di Aristotele, Politica 1333b)", [in] Polito - Talamo (a.c.), 2010, 131-155.

(2012): "Il ruolo delle staseis nella riflessione aristotelica sull'ordinamento politico di Sparta", [in] Polito - Talamo (a.c.), 2012, 69-93.

(2017): Sparta. Storia e rappresentazioni di una città greca, Roma.

Luraghi, N. (2008): The Ancient Messenians: Constructions of Ethnicity and Memory, Cambridge (http://dx.doi.org/10.1017/CBO9780511481413).

Luraghi, N. (ed.), (2001): The Historian's Craft in the Age of Herodotus, Oxford.

Maddoli, G. (1985): “'Attikà' prima di Erodoto?”, Studi Storici 7, 101-112.

Marincola, J. (ed.), (2007): A Companion to Greek and Roman Historiography, Malden (http://dx.doi.org/10.1002/9781405185110).

Mazzarino, S. (1966): Il pensiero storico classico, vol. 1 (=Biblioteca Universale Laterza. Collezione storica 91), Roma.

Meier, C. (1988): La nascita della categoria del politico in Grecia, Bologna.

Millender, E. G. (2001): "Spartan literacy revisited", Classical antiquity 20/1, 121-164 (http://dx.doi.org/10.1525/ca.2001.20.1.121).

Moggi, M. (2017): La polis e dintorni. Saggi raccolti in occasione del $75^{\circ}$ compleanno, Pisa.

Möller, A. (2001): "The beginning of chronography: Hellanicus' Hieraiai", [in] Luraghi (ed.), 2001, 241-262.

Momigliano, A. (1984): "Storia antica e antiquaria", [in] A. Momigliano (ed.), Sui fondamenti della storia antica, Torino, 3-45. 
(1991): “The Rise of Antiquarian Research", [in] A. Momigliano (ed.), The Classical Foundations of Modern Historiography, Berkeley-Los Angeles-London, 54-79.

Morgan, K. A. (2012): "Plato and the Stability of History", [in] J. Marincola - L. LlewellynJones - C. Maciver (eds.), Greek Notions of the Past in the Archaic and Classical Eras. History without Historians, Edimburgh, 227-252.

Mossé, C. (1978), "Le thème de la patrios politeia dans la pensée grecque du IVeme siècle", Eirene 16, 81-89.

Mulhern, J. J.

(2014): ПOЛITEIA in Aristotle's Politica: An Annotated Catalogue (retrieved from http:// repository.upenn.edu/classics_papers/31).

(2015): "Politeia in Greek Literature, Inscriptions and Aristotle's Politics: Reflections on translation and interpretation", [in] T. Lockwood - T. Samaras (eds.), Aristotle's Politics: A Critical Guide, Cambridge, 84-102 (https://doi.org/10.1017/ CBO9781107280748.006).

Munn, M. (2000): The School of History. Athens in the Age of Socrates, Berkeley-Los Angeles-London.

Murray, O.

(1993a): "Polis and Politeia in Aristotle", [in] M. H. Hansen (ed.), The Ancient Greek City-State, (=Historisk-filosofiske Meddelelser 67), Copenhagen, 197-210.

(1993b): La città greca (=Piccola biblioteca Einaudi 599), Torino.

Musti, D. (1995): Demokratia: origine di un'idea, Bari.

Nafissi, M.

(2009): "Sparta", [in] K. A. Raaflaub - H. van Wees (eds.), A Companion to Archaic Greece, Malden, 117-137 (http://dx.doi.org/10.1002/9781444308761.ch7).

(2010): “The Great Rhetra (Plu. Lyc. 6): A Retrospective and Intentional Construct?”, [in] Foxhall - Gehrke - Luraghi (eds.), 2010, 89-119.

Nicolai, R.

(2007): "The Place of History in the Ancient World", [in] Marincola (ed.), 2007, 13-26 (http://dx.doi.org/10.1002/9781405185110.ch1).

(2010): “L'attidografia come genere letterario", [in] Bearzot - Landucci (a.c.), 2010, 3-28.

Ober, J.

(1989): Mass and Elite in Democratic Athens: Rhetoric, Ideology, and the Power of the People, Princeton.

(2005): “Aristotle's Natural Democracy”, [in] R. Kraut - S. Skultety (eds.), Aristotle's Politics: Critical Essays, Lanham, 223-243.

Orsi, D. P. (1994): “La storiografia locale”, [in] G. Cambiano - L. Canfora - D. Lanza (a.c.), Lo spazio letterario della Grecia antica, Vol. I, T. III, Roma, 149-179.

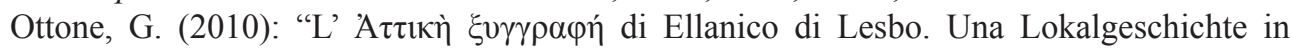
prospettiva eccentrica”, [in] Bearzot - Landucci (a.c.), 2010, 53-112.

Paradiso, A.

(1994): "Tucidide, Aristotele, la stasis a Sparta. Due modelli interpretativi”, Mètis 9-10, 151-170.

(1995): "Tempo della tradizione, tempo dello storico: Thuc. I. 18 e la storia arcaica spartana", Storia della Storiografia 28, 35-45.

(2000): "Lycurgue spartiate", [in] C. Darbo-Peschanski (ed.), Constructions du temps dans le monde grec, Paris, 373-391.

Poddighe, E.

(2014): Aristotele, Atene e le metamorfosi dell'idea democratica, Roma. 
(2016): "Giustizia e costituzione: scienza politica e intelligibilità della storia secondo Aristotele (EN, V, 1134a 25-1135a 8)", Gerión 34, 77-101 (http://dx.doi.org/10.5209/ rev_GERI.2016.v34.53736).

(2017): "La funzione della conoscenza storica nella teoria politica e nella precettistica retorica secondo Aristotele: l'importanza della visione globale", Nova Tellus 35/2, 61-81 (http://dx.doi.org/10.19130/iifl.nt.2017.35.2.768).

(2018a): “Arist. Ath. Pol. 9.2 e la regola del giudizio globale sui politika. Considerazioni sul metodo storico aristotelico" [in] Bearzot - Canevaro - Gargiulo - Poddighe (a.c.), 2018, 147-174.

(2018b): “Aristotele e la legge: il tema del mutamento", [in] B. Biscotti (ed.), Kállistos Nómos. Scritti in onore di Alberto Maffi (=Collana del Dipartimento Giurisprudenza dell'Università di Milano-Bicocca 129), Torino, 153-176.

(2019): Synoran. Studi sul pensiero storico e politico di Aristotele, Milano.

Polansky, R. (1991): “Aristotle on Political Change", [in] D. Keyt - F. D. Miller (eds.), A Companion to Aristotle's Politics, Oxford, 322-345.

Polito, M.

(2001): Dagli scritti di Eraclide sulle costituzioni: un commento storico (=Quaderni del Dipartimento di Scienze dell'Antichità dell'Università di Salerno 26), Napoli.

(2017): "Gli opuscoli sulla politeia della Scuola di Aristotele: appunti e riflessioni”, La Parola del Passato 71, 11-43.

Polito, M. - Talamo, C. (a.c.)

(2010): La politica di Aristotele e la storiografia locale, Tivoli.

(2012): Istituzioni e costituzioni in Aristotele tra storiografia e pensiero politico (=Themata 10), Tivoli.

Porciani, L.

(2001a): Prime forme della storiografia greca. Prospettiva locale e nazionale nella narrazione storica (=Historia Einzelschriften 152), Stuttgart.

(2001b): "La storia locale in Grecia secondo Dionigi di Alicarnasso", [in] C. Bearzot (a.c.), Storiografia locale e storiografia universale: forme di acquisizione del sapere storico nella cultura antica, Como, 287-297.

(2006), "Il problema della storia locale", [in] C. Ampolo (a.c.), Aspetti dell'opera di Felix Jacoby, Pisa, 173-184.

Pownall, F. (2004): Lessons from the Past. The Moral Uses of History in Fourth-Century Prose, Ann Arbor (http://dx.doi.org/10.3998/mpub.17777).

Raaflaub, K. A. (2010): "Ulterior Motives in Ancient Historiography: What Exactly and Why?", [in] Foxhall - Gehrke - Luraghi (eds.), 2010, 189-210.

Rhodes, P. J.

(1990): “The Attidographers”, [in] H. Verdin - G. Schepens - E. de Keyser (eds.), 1990, 73-81.

(2006): “'Classical' and 'Hellenistic' in Athenian History”, [in] E. Dąbrowa (ed.), Greek and Hellenistic Studies (=Electrum, Studies in Ancient History 11), Kraków, 27-43.

(2018): "What is a Constitution", [in] Bearzot - Canevaro - Gargiulo - Poddighe (a.c.), 2018, 21-31.

Riedinger, J. C. (1991): Étude sur les Helléniques, Paris.

Rood, T. (2012): "The plupast in Xenophon's Hellenika", [in] J. Grethlein - C. B. Krebs (eds.), Time and Narrative in Ancient Historiography. The 'Plupast' from Herodotus to Appian, Cambridge, 76-94 (http://dx.doi.org/10.1017/CBO9781139035200.006). 
Russo, L. (2013): La rivoluzione dimenticata. Il pensiero scientifico greco e la scienza moderna, Milano.

Sancho Rocher, L.

(1994): “Stásis y krâsis en Tucídides (8. 97.1-2)”, Habis 25, 41-70.

(2004): “Los 'moderados' atenienses y la implantación de la oligarquía. Corrientes políticas en Atenas entre el 411 y 403 a. C.", Veleia 21, 73-98.

(2007): "Athenaion Politeia 34.3, about Oligarchs, Democrats and Moderates in the Late Fifth Century BC", Polis: The Journal of the Society for Greek Political Thought 24.2, 298-327.

Saxonhouse, A. W. (1996): Athenian Democracy: Modern Mythmakers and Ancient Theorists, London.

Schepens, G.

(2001): “Ancient Greek City Histories. Self-Definition through History Writing”, [in] K. Demoen (ed.), The Greek City from Antiquity to the Present: Historical Reality, Ideological Construction, Literary Representation, Louvain-Paris, 3-25.

(2006), "Travelling Greek Historians", [in] M. G. Angeli Bertinelli - A. Donati (a.c.), Le vie della storia. Migrazioni di popoli, viaggi di individui, circolazione di idee nel Mediterraneo antico. Atti del II Incontro internazionale di storia antica (Genova 6-8 ottobre 2004), (=Serta antiqua et mediaevalia 9. Storia Antica 5), Roma, 81-102.

Schofield, M. (2006): Plato: Political Philosophy, Oxford.

Strauss, L. (1964): The City and Man, Chicago.

Susanetti, D. (2015): Tucidide. I discorsi della democrazia, Milano.

Thomas, R.

(1994): "Law and Lawgiver in the Athenian Democracy", [in] R. Osborne - S. Hornblower (eds.), Ritual, Finance, Politics. Athenian Democratic Accounts Presented to D. Lewis, Oxford, 119-133.

(2011): "Thucydides and Social Change: Between Akribeia and Universality", [in] Lianeri (ed.), 2011, 229-246 (http://dx.doi.org/10.1017/CBO9780511975998.011).

(2014a): "The Greek Polis and the Tradition of Polis History: Local History, Chronicles and the Patterning of the Past", [in] A. Moreno - R. Thomas (eds.), Patterns of the Past. Epitédeumata in the Greek Tradition, Oxford, 145-172 (http://dx.doi.org/10.1093/ acprof:osobl/9780199668885.003.0008).

(2014b): "Local History, Polis History, and the Politics of Place", [in] G. Parmeggiani (ed.), Between Thucydides and Polybius. The Golden Age of Greek Historography (=Hellenic Studies Series 64), Washington D.C., 239-262.

(2019): Polis Histories. Collective Memories and the Greek World, Cambridge (https:// doi.org/10.1017/9781108147897).

Tober D.

(2010): "Politeiai and Spartan Local History", Historia 59/4, 412-431.

(2017): “Greek Local Historiography and its Audiences”, Classical Quarterly 67/2, 460 484 (http://dx.doi.org/10.1017/S0009838817000519).

Toye, D. L. (1999): “Aristotle's Other Politeiai: Was the Athenaion Politeia Atypical?", Classical Journal 94/3, 235-253.

Vartsos, I. A. (2008): "Fifth-Century Athens: Citizens and Citizenship", Parnassos 50, 65-74.

Verdin, H. - Schepens, G. - Keyser, E. de (eds.), (1990): Purposes of History. Studies in Greek Historiography from the 4th to the 2nd centuries B.C. (=Studia Hellenistica 30), Leuven.

Wallace, R. W. (1989), The Areopagos Council to 307 B.C., Baltimore.

Walters, K .R. (1976): “The Ancestral Constitution and Fourth-Century Historiography in Athens", American Journal of Ancient History 1, 129-144. 